\title{
Time-Optimal Cooperative Path Tracking for Multi-Robot Systems
} Hamed Haghshenas 



\section{Time-Optimal Cooperative Path Tracking for Multi-Robot Systems}

\section{Hamed Haghshenas}

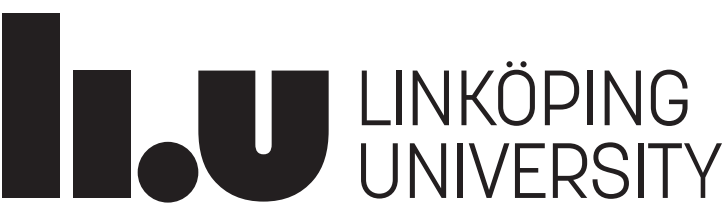


This is a Swedish Licentiate's Thesis.

Swedish postgraduate education leads to a Doctor's degree and/or a Licentiate's degree.

A Doctor's Degree comprises 240 ECTS credits (4 years of full-time studies).

A Licentiate's degree comprises 120 ECTS credits,

of which at least 60 ECTS credits constitute a Licentiate's thesis.

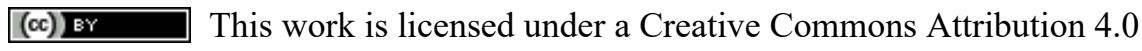
International License.

https://creativecommons.org/licenses/by/4.0

Linköping studies in science and technology. Licentiate Thesis

No. 1915

Time-Optimal Cooperative Path Tracking for Multi-Robot Systems

Hamed Haghshenas

hamed.haghshenaseliu.se

www. control.isy.liu.se

Department of Electrical Engineering

Linköping University

SE-581 83 Linköping

Sweden

ISBN 978-91-7929-014-6ＩSSN 0280-7971

Copyright (C) 2021 Hamed Haghshenas

Printed by LiU-Tryck, Linköping, Sweden 2021 
To my family! 



\section{Abstract}

Robotic systems are nowadays the key technology in a wide variety of applications. The increasing demand for performance of robotic systems is often met by employing a team of cooperating robots for a specific task. When the task carried out by the robots involves manipulation of an object, the multi-robot system is said to perform a cooperative manipulation task. Cooperative manipulation is an important capability for extending the domain of robotic applications. This thesis studies the time-optimal path tracking problem for a cooperative manipulation scenario where an object is rigidly grasped by multiple manipulators. The goal is to move the object along a predefined geometric path in minimum time while satisfying the imposed constraints on the motion. First, it is shown that the time-optimal path tracking problem for cooperative manipulators can be cast as a convex optimization problem. A fundamental property of convex optimization problems is that any locally optimal solution is also a globally optimal one. Furthermore, by recognizing and formulating a problem as a convex optimization problem, it can be solved very reliably and efficiently using interior-point or other methods for convex optimization. These results are presented in two separate studies. In the first one which is a preliminary study, the manipulation setup is a particular setup comprised of two planar manipulators and a bar. Furthermore, the load distribution among the manipulators is considered to be equal. The second study extends the results in the preliminary study to a general scenario with $N$ generic manipulators and an object with a desired orientation during the motion. Here, the load distribution among the manipulators is determined via a generic pseudo-inverse of the grasp matrix that can be chosen by the user. The freedom in the choice of the pseudo-inverse allows to consider different load distributions which can be exploited to account for the potential differences in the capabilities of the manipulators. The second part of this thesis is devoted to finding load distributions that are free of internal forces. A drawback of using multiple manipulators in a cooperative manipulation task is that internal forces can be introduced. Internal forces are forces exerted by the end-effectors at the grasping points that do not contribute to the motion of the manipulated object. While a certain amount of such forces can be useful in some cases, in general they must be avoided to prevent object damage and unnecessary effort of the manipulators. This thesis proposes a new approach to obtain internal force-free load distributions. The proposed approach results in a new pseudo-inverse of the grasp matrix parameterized by coefficients that have the meaning of the inertial parameters of some parts of the object. The freedom in the choice of the parameters of the pseudo-inverse allows to assign different loads to the manipulators. This can be exploited to account for the differences in the power capabilities of the manipulators. The results are further explored for scenarios where the object is three-dimensional and convex and has uniform mass density. Finally, the proposed pseudo-inverse is combined with the results in the first part of the thesis to solve the problem of time-optimal cooperative path tracking subject to zero internal forces during the motion. 



\section{Populärvetenskaplig sammanfattning}

Robotsystem är idag vanliga inom tillverkningsindustri, logistik, byggindustri, jordbruk, övervakning, livräddning och i olika servicetillämpningar. Ökade krav på prestanda och tillförlitlighet uppfylls inte sällan genom att använda flera robotar för att tillsammans lösa ett problem. Flera samarbetande robotar löser problem som en enskild robot inte klarar av, precis som en människa med två armar kan klara av att lösa problem som en människa med bara en arm inte klarar. En vanlig tillämpning är att hålla och transportera stora och/eller tunga objekt. För att lösa detta problem måste robotarna klara av att se till att objektet följer en på förhand specificerad bana, i många fall är det också viktigt att banan följs så snabbt som möjligt, d.v.s. på minimal tid. Andra relevanta kriterier kan vara att använda så lite energi som möjligt vid förflyttningen. Det är därför av intresse att optimera dessa kriterier så att robotarnas fulla potential utnyttjas maximalt. Denna avhandling studerar tidsoptimal banföljning för samarbetande robotar. I första delen av avhandlingen visas hur detta problem kan formuleras som ett konvext optimeringsproblem. Fördelen med detta är att det finns mycket effektiva och tillförlitliga numeriska beräkningsmetoder för denna klass av problem. Andra delen av avhandlingen studerar hur robotarna kan samverka för att lösa uppgiften utan att tillföra krafter på objektet som ska flyttas som bidrar till förflyttningen, d.v.s. krafter som orsakar extra spänningar i objektet. Dessa krafter är ibland av godo, men ofta är det tvärtom, eftersom de kan skada objektet, och dessutom så kan de orsaka onödig energiförbrukning i robotarna. I denna avhandling föreslås en ny metod för att undvika dessa krafter vid banföljning för samarbetande robotar. 



\section{Acknowledgments}

First and foremost, I would like to thank my supervisor Prof. Anders Hansson and my co-supervisor Prof. Mikael Norrlöf for the guidance and support over the past years. Thank you for all the feedback, encouragement, and for always being available for discussions. You have been great sources of ideas and inspiration.

I would also like to thank former head of division Prof. Svante Gunnarsson, and current head of division Assoc. Prof. Martin Enqvist for maintaining a friendly and professional work environment, and Ninna Stensgård for kindly helping me with administrative tasks.

To all my current and former colleagues at the division, thank you for contributing to a nice and friendly atmosphere. The group at Automatic Control is truly great, and I appreciate being part of it. Special thanks to Lic. Fredrik Ljungberg, Lic. Daniel Arnström, M.Sc. Shamisa Shoja, and Lic. Magnus Malmström for their help in proofreading parts of this thesis.

I also want to acknowledge the financial support from the Excellence Center at Linköping-Lund in Information Technology (ELLIIT) and the Vinnova Competence Center LINK-SIC which made the research presented in this thesis possible.

Finally, I would like to thank my family for their love and unconditional support.

Linköping, September 2021

Hamed Haghshenas 



\section{Contents}

Notation

xiii

1 Introduction 1

1.1 Research motivation . . . . . . . . . . . . . . . . 1

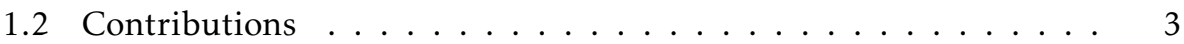

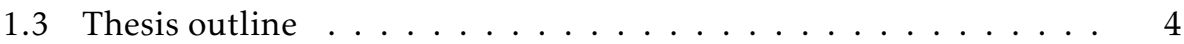

2 Background $5 \ldots \ldots \ldots$

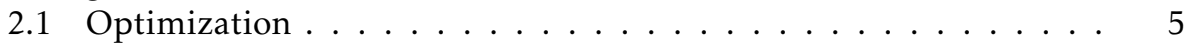

2.1 .1 Convex optimization $\ldots \ldots \ldots \ldots \ldots \ldots$

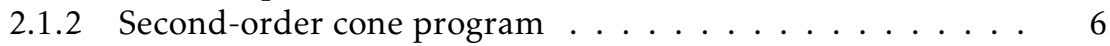

2.2 Manipulator dynamics . . . . . . . . . . . . . 6

3 Optimal path tracking for robot manipulators 9

3.1 Optimal motion planning for robots $\ldots \ldots \ldots \ldots$

3.2 Time-optimal path tracking $\ldots \ldots \ldots \ldots \ldots$

3.2 .1 Related work . . . . . . . . . . . . . . . . . 10

3.2.2 Original problem formulation . . . . . . . . . . . 11

3.2.3 Convex formulation . . . . . . . . . . . . . . . . 12

3.2.4 Numerical approach . . . . . . . . . . . . . . . 13

3.2.5 Second-order cone program formulation . . . . . . . . 14

4 Preliminary developments 17

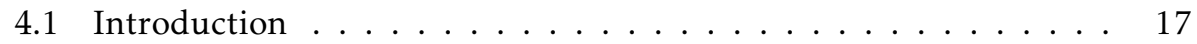

4.2 Problem statement . . . . . . . . . . . . . . . 18

4.3 Simplified scenario . . . . . . . . . . . . . . . . 18

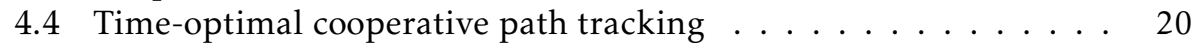

4.5 Numerical simulation . . . . . . . . . . . . . . . . . . . . 24

5 General framework for time-optimal cooperative path tacking 29

5.1 Problem statement . . . . . . . . . . . . . . . . . 29

5.1 .1 Kinematics . . . . . . . . . . . . . . . . . 30

5.1 .2 Manipulator dynamics . . . . . . . . . . . . 31 


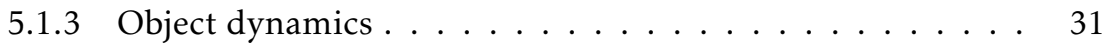

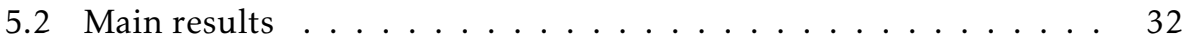

5.3 Numerical simulation . . . . . . . . . . . . . . 35

6 Internal force-free cooperative path tracking 41

6.1 Introduction . . . . . . . . . . . . . . . . 41

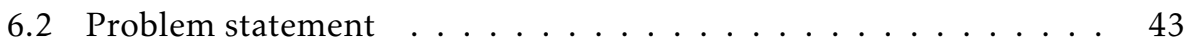

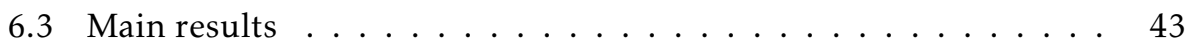

6.4 Numerical simulation . . . . . . . . . . . . . . . 50

7 Concluding remarks $\quad 57$

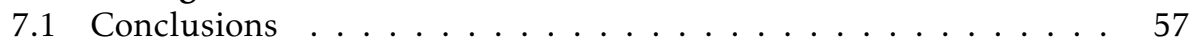

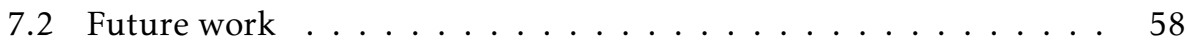

$\begin{array}{ll}\text { Bibliography } & 61\end{array}$ 
Notation

SETS

\begin{tabular}{cl}
\hline Notation & Meaning \\
\hline $\mathbb{R}^{n}$ & Set of real column vectors of dimension $n$ \\
$\mathbb{R}^{n \times m}$ & Set of real $n \times m$ matrices \\
\hline
\end{tabular}

\section{SymbOLS AND OPERATORS}

\begin{tabular}{cl}
\hline Notation & Meaning \\
\hline$I_{n}$ & Identity matrix of dimension $n \times n$ \\
$0_{n \times m}$ & $n \times m$ matrix with all its elements equal to zero \\
$0_{n}$ & Column vector of dimension $n$ with all entries equal \\
& to zero \\
$S(\cdot)$ & Skew-symmetric operator \\
$\operatorname{diag}\left(A_{1}, \ldots, A_{N}\right)$ & Block diagonal matrix with $A_{1}, \ldots, A_{N}$ on the diagonal \\
$\|x\|$ & Euclidean norm of vector $x$ \\
\hline
\end{tabular}

\section{Abbreviations}

\begin{tabular}{cl}
\hline Abbreviation & Meaning \\
\hline SOCP & Second-order cone program \\
DOF & Degrees of freedom \\
\hline
\end{tabular}





\section{1}

Introduction

In this thesis, the problem of time-optimal path tacking for a team of cooperating robotic manipulators is studied. This introductory chapter gives the motivation behind this problem, lists the contributions, and outlines the content of the thesis.

\subsection{Research motivation}

Robotic systems are nowadays the key technology in a wide range of application domains, from construction, manufacturing and agriculture to search and rescue, and service robotics. The increasing demand for performance of robotic systems is often met by using multiple robots for a specific task. A team of cooperating robots outperforms the functionality of a single robot; like a human being using two arms has an advantage over one using only one arm. When the task conducted by multiple robots involves manipulation of an object, the multirobot system is said to perform a cooperative manipulation task. Cooperative manipulation is an important capability for extending the domain of robotic applications. Typical examples include industrial manipulators manipulating large or heavy objects as well as transportation tasks conducted by multiple robots. See Figure 1.1 for some examples of cooperative manipulation. One particular subject that most of these applications have in common is path tracking. Path tracking is the second stage of the so-called decoupled approach (Choset et al. [2005]; LaValle [2006]); an approach for solving motion planning problems. The first stage of the decoupled approach, known as path planning, determines a path while taking geometric aspects of the task and the environment into account, whereas the path tracking stage is concerned with the dynamic aspects of the robot. In many robotic applications, the path is determined by the task and its specifications. Hence, assuming that a desired geometric path is given, 
the path tracking problem can be studied independently and is of value on its own.

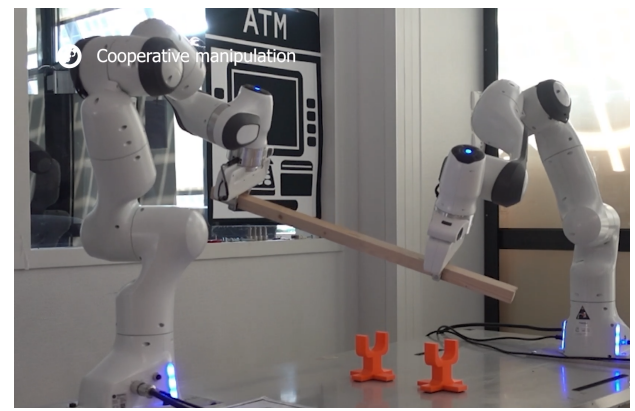

(a) Cooperative manipulation performed by two Panda robots (PRO).

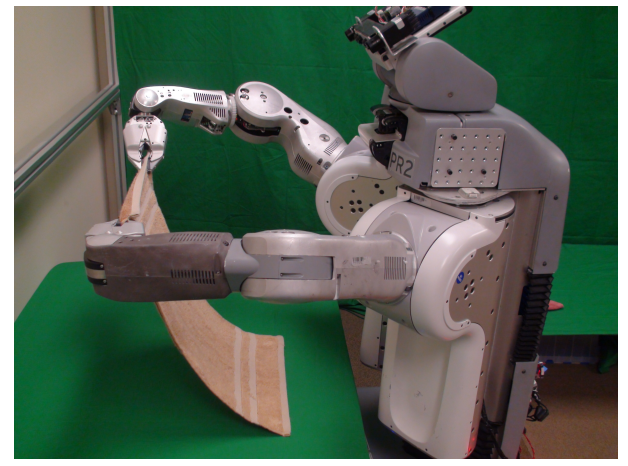

(c) PR2 robot folding a towel (MaitinShepard et al. [2010]).

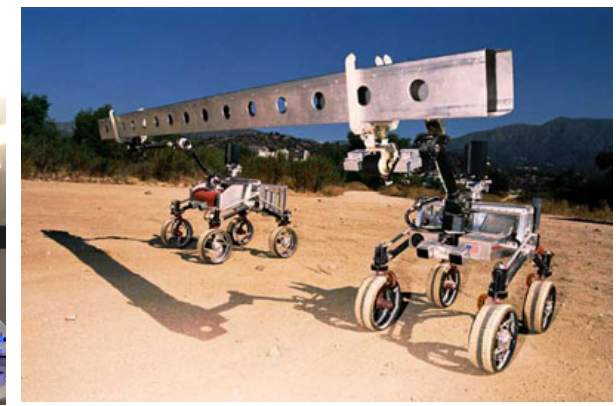

(b) Two NASA rovers handling and transporting a large beam (TRN [2002]).

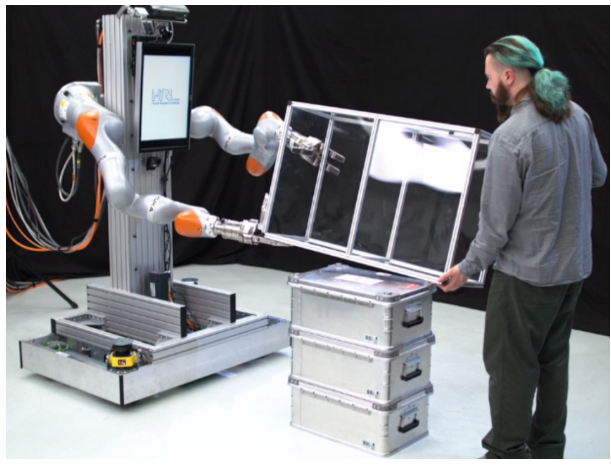

(d) Human-robot cooperative object manipulation (Gienger et al. [2018]).

Figure 1.1: Examples related to cooperative manipulation.

For a wide range of robotic applications, it is desired to minimize the execution time of a task or some other criteria such as the energy consumed during the motion. For example, robotic manipulators performing a variety of tasks are included in almost every production line, and there is an obvious relation between the execution time of the tasks and productivity. Hence, time-optimal and in general optimal motion planning are of great significance for robotic systems. By 
optimizing robots motions while taking their dynamics into account, robots can fully exploit their capabilities.

Motivated by these observations, this thesis studies the time-optimal path tracking problem for a team of cooperating robotic manipulators. We consider a scenario where an object is rigidly grasped by multiple manipulators. Timeoptimal cooperative path tracking addresses the problem of moving the object along a predefined geometric path in minimum time, which requires realizing a velocity as high as possible along the path while satisfying the imposed constraints on the motion. When dealing with this problem, some issues need to be taken into consideration that normally do not arise in path tracking for a single manipulator. These issues are related to the presence of an object and multiple manipulators and the interplay between them. Among these issues are the imposed kinematic constraints on the whole system, the incorporation of the object dynamics, the load distribution among the manipulators, and the role of internal forces.

A drawback of using multiple manipulators in a cooperative manipulation task is that internal forces can be introduced. In general, there exists an infinite number of generalized forces exerted by the end-effectors that result in the same desired generalized forces on the object in a cooperative manipulation task. This is due to multiple manipulators being involved. Normally, this redundancy is resolved using a load distribution strategy and by means of a pseudo-inverse. The resulting interaction forces among the manipulators and the object are decomposed into motion-inducing and internal forces. Internal forces are forces exerted by the end-effectors at the grasping points that do not contribute to the motion of the manipulated object. While a certain amount of such forces can be useful in some cases (e.g., in order to ensure a firm grasp), in general they must be avoided to prevent object damage and unnecessary effort of the manipulators. In most works that have focused on characterizing internal forces, e.g., in Verginis et al. [2019], unique load distributions are introduced as a load distribution free of internal forces. In contrast to these results, the work by Erhart and Hirche [2015] proposes a parameterized pseudo-inverse for this purpose, suggesting that the load distributions free of internal forces are not unique and there can be infinitely many load distributions of this type. The work by Erhart and Hirche [2015] finds some of these load distributions. It can be said that finding all load distributions that are free of internal forces is still an open problem. Motivated by this and considering that internal forces can appear in the path tracking problem for cooperative manipulators, a chapter in this thesis is devoted to finding some other internal force-free load distributions, where a novel approach is proposed for this purpose.

\subsection{Contributions}

The contributions of this thesis are presented in Chapters 4, 5 and 6 and are based on published and unpublished material. The content of Chapter 4 is based on the following publication: 
Hamed Haghshenas, Mikael Norrlöf, and Anders Hansson. A convex optimization approach to time-optimal path tracking problem for cooperative manipulators. IFAC-PapersOnLine, 52(10):400-405, 2019.

A preliminary study of the time-optimal cooperative path tracking problem is presented in this paper. A particular setup comprised of two planar manipulators and an object is considered, and it is shown that the time-optimal path tracking problem for this setup can be formulated as a convex optimization problem. A fundamental property of convex optimization problems is that any locally optimal solution is also a globally optimal one. Furthermore, by recognizing and formulating a problem as a convex optimization problem, it can be solved very reliably and efficiently using interior-point or other methods for convex optimization.

In Chapter 5, the results in Chapter 4 are extended to a general scenario with $N$ generic manipulators and an object with a desired orientation during the motion. The proposed extension formulates the time-optimal path tracking problem for this general scenario as a convex optimization problem. Besides, a generic pseudo-inverse of the grasp matrix is used to determine the distribution of the load among the manipulators, whereas in Chapter 4 an equal load distribution is considered. This allows to account for the potential differences in the capabilities of the manipulators.

Chapter 6 proposes a new approach to obtain load distributions free of internal forces. The result is a new pseudo-inverse of the grasp matrix parameterized by coefficients that have the meaning of the inertial parameters of some parts of the object. Using this parameterized pseudo-inverse leads to internal force-free distributions. The freedom in the choice of the parameters of the pseudo-inverse allows for different load distribution, all free of internal forces, and to account for the differences in the power capabilities of the manipulators. The results are further explored for scenarios where the object is three-dimensional and convex and has uniform mass density.

\subsection{Thesis outline}

The remainder of the thesis is organized as follows: In Chapter 2, the necessary background material from mathematical optimization and dynamic models of robotic manipulators are reviewed. Chapter 3 gives an introduction to optimal motion planning and presents some known results on the problem of time-optimal path tracking for a single manipulator. Chapter 4 shows how the time-optimal path tracking problem for a particular scenario with cooperative manipulators holding a bar can be cast as a convex optimization problem. We consider this as a preliminary study of the time-optimal cooperative path tracking problem, which extends to a general scenario in Chapter 5 to account for generic manipulators and objects. In Chapter 6, an approach to obtain internal force-free load distributions is proposed. Finally, Chapter 7 concludes the thesis and discusses some ideas for future work. 


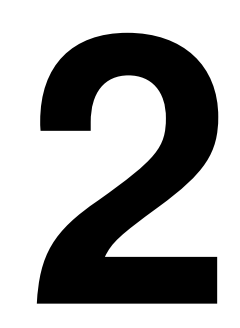

\section{Background}

This chapter provides the theoretical background required for the forthcoming chapters. The chapter starts with a review of mathematical optimization, convex optimization and second-order cone programs and continues with dynamic model for manipulators.

\subsection{Optimization}

Let us use the notation

$$
\begin{array}{cl}
\underset{z}{\operatorname{minimize}} & f_{0}(z) \\
\text { subject to } & f_{i}(z) \leq 0, \quad i=1, \ldots, v, \\
& f_{i}(z)=0, \quad i=v+1, \ldots, v+w,
\end{array}
$$

to describe the problem of finding a $z$ that minimizes $f_{0}(z)$ among all $z$ that satisfy the constraints $f_{i}(z) \leq 0, i=1, \ldots, v$, and $f_{i}(z)=0, i=v+1, \ldots, v+w$. Here, $z \in \mathbb{R}^{r}$ is the optimization variable, $f_{0}: \mathbb{R}^{r} \rightarrow \mathbb{R}$ is the objective function, $f_{i}: \mathbb{R}^{r} \rightarrow \mathbb{R}$ for $i=1, \ldots, v$, are the inequality constraint functions, and $f_{i}: \mathbb{R}^{r} \rightarrow \mathbb{R}$ for $i=v+1, \ldots, v+w$, are the equality constraint functions. The domain $\mathcal{D} \subseteq \mathbb{R}^{r}$ of the optimization problem (2.1) is the set of all points where the objective and all constraint functions are defined. A point $z \in \mathcal{D}$ is said to be feasible if it satisfies the constraints $f_{i}(z) \leq 0, i=1, \ldots, v$, and $f_{i}(z)=0, i=v+1, \ldots, v+w$. If there exists at least one feasible point, the optimization problem (2.1) is said to be feasible, and infeasible otherwise. 


\subsubsection{Convex optimization}

Convex optimization is a class of optimization problems that has great advantages and is useful in many fields of science and engineering. The most basic advantage to recognize or formulate a problem as a convex optimization problem is that it can then be solved very reliably and efficiently, using interior-point methods or other special methods for convex optimization (Boyd et al. [2004]). Before reviewing the definition of a convex optimization problem, we present some auxiliary definitions that are required for characterizing such problems.

Definition 2.1 (Convex set). A set $\mathcal{C}$ is convex if the line segment between any two points in $\mathcal{C}$ lies in $\mathcal{C}$, i.e., if for any $z_{1}, z_{2} \in \mathcal{C}$ and any $\theta \in[0,1]$ it holds that

$$
\theta z_{1}+(1-\theta) z_{2} \in \mathcal{C}
$$

Definition 2.2 (Convex function). A function $f: \mathbb{R}^{r} \rightarrow \mathbb{R}$ is convex if its domain $\mathcal{D} \subseteq \mathbb{R}^{r}$ is a convex set and if for all $z_{1}, z_{2} \in \mathcal{D}$ and any $\theta \in[0,1]$ it holds that

$$
f\left(\theta z_{1}+(1-\theta) z_{2}\right) \leq \theta f\left(z_{1}\right)+(1-\theta) f\left(z_{2}\right)
$$

Having defined convex sets and functions, we can now define a convex optimization problem. Consider the optimization problem given in (2.1). This problem is referred to as convex optimization problem if the objective function $f_{0}$ and the inequality constraint functions $f_{i}$ for $i=1, \ldots, v$, are all convex functions and the equality constraint functions $f_{i}$ for $i=v+1, \ldots, v+w$, are affine, i.e., $f_{i}(z)=a_{i}^{T} z-b_{i}$, for $i=v+1, \ldots, v+w$ (Boyd et al. [2004]).

\subsubsection{Second-order cone program}

Second-order cone programs (SOCP) are a subclass of convex optimization problems and can be solved almost as easily as linear programs using the methods such as interior-point methods (Boyd et al. [2004]). An SOCP has the following form:

$$
\begin{array}{ll}
\underset{z}{\operatorname{minimize}} & f^{T} z \\
\text { subject to } & \left\|A_{i} z+b_{i}\right\|_{2} \leq c_{i}^{T} z+d_{i}, \quad i=1, \ldots, v, \\
& F z=e,
\end{array}
$$

where $z \in \mathbb{R}^{r}$ is the optimization variable, $A_{i} \in \mathbb{R}^{r_{i} \times r}$, and $F \in \mathbb{R}^{k \times r}$.

\subsection{Manipulator dynamics}

The dynamic model of a manipulator provides a description of the relationship between its motion and the joint torques. Dynamic models are important for 
simulation of motion, testing motion planning techniques, and design of control algorithms.

One of the methods for deriving the equations of motion of a manipulator in the joint space is based on the Lagrange formulation. The Lagrangian of a mechanical system is defined as the difference between its kinetic and potential energies. That is,

$$
\mathcal{L}(q, \dot{q})=\mathcal{T}(q, \dot{q})-\mathcal{U}(q),
$$

where $\mathcal{T}$ and $\mathcal{U}$ denote the kinetic and potential energies of the system, respectively, and $q$ is the vector of generalized coordinates. A system with $n$ degrees of freedom can be described by $n$ generalized coordinates $q=\left[\bar{q}_{1}, \ldots, \bar{q}_{n}\right]^{T}$. The equations of motion are given by the Euler-Lagrange equations (see e.g., Siciliano et al. [2010])

$$
\frac{d}{d t} \frac{\partial \mathcal{L}(q, \dot{q})}{\partial \dot{\bar{q}}_{i}}-\frac{\partial \mathcal{L}(q, \dot{q})}{\partial \bar{q}_{i}}=\bar{\tau}_{i},
$$

where $\bar{\tau}_{i}$ is the generalized force associated with $\bar{q}_{i}$. For a robotic manipulator, the generalized coordinates are the joint variables and the generalized forces are the corresponding joint torques. For a manipulator with $n$ degrees of freedom (DOF) and joint variables $q \in \mathbb{R}^{n}$, the resulting equations from (2.6) can be written in matrix form as

$$
M(q) \ddot{q}+C(q, \dot{q}) \dot{q}+g(q)=\tau,
$$

where $\tau \in \mathbb{R}^{n}$ are the joint torques, $M(q) \in \mathbb{R}^{n \times n}$ is the positive definite inertia matrix, $C(q, \dot{q}) \in \mathbb{R}^{n \times n}$ is the matrix of Coriolis and centrifugal forces, and $g(q) \in$ $\mathbb{R}^{n}$ is the vector of gravitational terms.

In a scenario with more that one manipulator, we will add the subindex $i$ to refer to the $i$ th manipulator. So, the equation describing the dynamics of the $i$ th manipulator with $n_{i}$ DOF and joint variables $q_{i} \in \mathbb{R}^{n_{i}}$ becomes

$$
M_{i}\left(q_{i}\right) \ddot{q}_{i}+C_{i}\left(q_{i}, \dot{q}_{i}\right) \dot{q}_{i}+g_{i}\left(q_{i}\right)=\tau_{i},
$$

where $\tau_{i} \in \mathbb{R}^{n_{i}}$ are the joint torques, $M_{i}(q) \in \mathbb{R}^{n_{i} \times n_{i}}$ is the inertia matrix, $C_{i}(q, \dot{q}) \in$ $\mathbb{R}^{n_{i} \times n_{i}}$ is the matrix of Coriolis and centrifugal forces, and $g_{i}(q) \in \mathbb{R}^{n_{i}}$ is the vector of gravitational terms.

If the $i$ th manipulator's end-effector is in contact with an environment, a portion of the actuation torques is used to balance the torques induced at the joints by the contact forces. Such torques are given by $J_{i}^{T}\left(q_{i}\right) h_{i}$ where $J_{i} \in \mathbb{R}^{6 \times n_{i}}$ is the $i$ th manipulator's geometric Jacobian, and $h_{i} \in \mathbb{R}^{6}$ is the vector of generalized forces exerted by the $i$ th end-effector on the environment. The equations of motion for the $i$ th manipulator are then given by

$$
M_{i}\left(q_{i}\right) \ddot{q}_{i}+C_{i}\left(q_{i}, \dot{q}_{i}\right) \dot{q}_{i}+g_{i}\left(q_{i}\right)=\tau_{i}-J_{i}^{T}\left(q_{i}\right) h_{i} .
$$





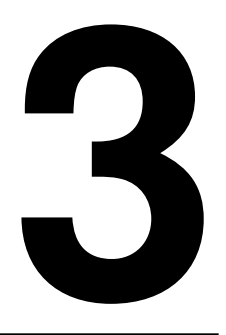

\section{Optimal path tracking for robot manipulators}

The purpose of this chapter is to collect some known results about time-optimal path tracking for a single manipulator that will be used in the rest of the thesis. The chapter starts with an introduction to optimal motion planning and continues by mentioning some related works on the time-optimal path tracking problem. Then in the subsequent sections, the original and convex formulation of the problem, together with the corresponding numerical solution are presented. Finally, an SOCP formulation of the problem is provided.

\subsection{Optimal motion planning for robots}

The goal of optimal robot motion planning is to find the trajectory in the joint or the operational space coordinates, to move from an initial posture to a final assigned posture, while not colliding obstacles. This transition optimizes some optimality criterion such as time or energy and must respect the actuator limits. Motion planning problems which only consider kinematic constraints such as bounds on velocity and acceleration are called kinematic motion planning. As a result of neglecting or simplification of the nonlinear robot dynamics, the obtained solution for these problems is suboptimal. On the other hand, some motion planning problems take the dynamics of the robot as well as its actuators' limits into account and are called dynamic motion planning. In this thesis, we are concerned with the dynamic motion planning rather than the kinematic.

In general, solving the dynamic motion planning problem is a difficult and complex task. This is because both the geometric path, considering obstacle avoidance and other geometric constraints, and the timing law on the path, considering system dynamics and actuator constraints, have to be taken into account and optimized. Consequently, instead of solving the motion planning problem in its entirety and directly in the system's state space, referred to as the direct 
approach (Choset et al. [2005]), the motion planning problem is decoupled to a path planning and a path tracking stage. This approach is called the decoupled approach (Choset et al. [2005]) and requires lower computational effort. The path planning stage determines a geometric path while taking account of task specifications, obstacle avoidance, other robots in a shared workspace, and other geometric aspects. The path tracking stage, which is the focus of this thesis, concerns itself with determining the optimal trajectory along a prescribed geometric path which is generated in the path planning stage, while taking the robot dynamics and the actuator constraints into account. It must however be noted that since in the decoupled approach the redundancy of the robot in the path tracking stage is lost by planning the path beforehand in the path planning stage, the resulting solution is suboptimal compared to the direct approach.

\subsection{Time-optimal path tracking}

Path tracking is also of value on its own since for a wide range of robotic applications the geometric path is determined by the task and its specifications. Path tracking problems whose optimality criterion is time are referred to as timeoptimal path tracking problems. In this section, we collect some known results regarding the time-optimal path tracking problem for a single robot manipulator. Before proceeding to state these results, we report some previous works that have focused on this problem.

\subsubsection{Related work}

There are different methods for solving the time-optimal path tracking problem, some of which exploit the fact that the motion along a predetermined path can be described by a single parameter, called path coordinate and denoted by $s$, and its time derivatives (Shin and McKay [1985]; Bobrow et al. [1985]; Verscheure et al. [2009]). In Shin and McKay [1985], Bobrow et al. [1985] and Constantinescu and Croft [2000], which fall into this category, the authors make use of the phase plane of $(s, \dot{s})$ in order to develop algorithms that construct the optimal trajectory. To minimize the traversal time, the acceleration along the path always takes either its largest or its smallest possible value. Therefore, finding the optimal trajectory amounts to finding the positions on the phase plane at which the acceleration switches between its maximum and minimum values.

While the early implementations have focused on iterative and geometric methods to find the optimal velocity profile, in Verscheure et al. [2009], it is shown that by exploiting a nonlinear change of variables, the time-optimal path tracking problem for a robotic manipulator can be cast as a convex optimization problem. This allows for efficiently solving the problem by utilizing the wide variety of algorithms and software developed for convex optimization (Boyd et al. [2004]). Furthermore, the convexity of the problem guarantees that any locally optimal solution is also a globally optimal one. It must however be noted that the change of variables used in Verscheure et al. [2009] has been known since at 
least 1985 (see e.g., Bobrow et al. [1985]; Dubowsky et al. [1986]; Pfeiffer and Johanni [1987]), although the resulting convexity of the problem was not noted back then.

Because of the advantages that the convex formulation offers, much work has been conducted to extend the range of applicability of these ideas to other problems such as path tracking for different type of vehicles studied in Lipp and Boyd [2014], or to include new types of constraints in the robot path tracking problem (Ardeshiri et al. [2011]; Reynoso-Mora et al. [2013]; Debrouwere et al. [2013]). Similarly as in Pfeiffer and Johanni [1987], viscous friction which renders the problem nonconvex is not considered in the robot model in Verscheure et al. [2009]. In this context, some works such as Ardeshiri et al. [2011] and Debrouwere et al. [2013] have addressed the incorporation of the constraints that destroy the convexity of the problem. The work Ardeshiri et al. [2011] incorporates speed dependent constraints into the problem and replaces the nonconvex constraints with their convex approximations to preserve the convexity of the problem, whereas the work Debrouwere et al. [2013] considers several robotic applications that result in nonconvex problems and employs sequential convex programming to solve the corresponding problem by writing the nonconvex constraints as differences of convex functions.

The change of variables introduced in Verscheure et al. [2009] is also used in other studies. In Steinhauser and Swevers [2018], a two-step iterative learning algorithm for path tracking problem of a robotic manipulator is proposed which compensates for a possible model-plant mismatch and improves the tracking performance. The work Debrouwere and Swevers [2016] studies the potential of adding counterweights to the robot structure in order to decrease the motion time along a given path.

\subsubsection{Original problem formulation}

Consider a prescribed path $q(s)$, given as a function of a scalar path coordinate $s$ in the joint space coordinates. If the path is given in the operational space coordinates, inverse kinematics procedures can be used to obtain the corresponding path in the joint space coordinates. The aim is to determine the timing along the path by minimizing the time as the objective function, subject to system dynamics and actuator constraints. The time dependency of the path is determined by the relation $s(t)$ between the path coordinate $s$ and time $t$. Without loss of generality, assume that the trajectory starts at $t=0$, ends at $t=T$, and that $s(0)=0 \leq s(t) \leq s(T)=1$. Furthermore, it is assumed that $\dot{s}(t) \geq 0, \forall t \in[0, T]$. In other words, we always move forward along the path. For the given path, the joint velocities and accelerations using the chain rule can be written as

$$
\begin{aligned}
& \dot{q}(s(t))=q^{\prime}(s(t)) \dot{s}(t), \\
& \ddot{q}(s(t))=q^{\prime}(s(t)) \ddot{s}(t)+q^{\prime \prime}(s(t)) \dot{s}(t)^{2},
\end{aligned}
$$

where $q^{\prime}(s)=\partial q(s) / \partial s, q^{\prime \prime}(s)=\partial^{2} q(s) / \partial s^{2}, \dot{s}(t)=d s / d t$ and $\ddot{s}(t)=d^{2} s / d t^{2}$. Substituting these expressions for $\dot{q}(s(t))$ and $\ddot{q}(s(t))$ in $(2.7)$, yields the following ex- 
pression for the equations of motion

$$
\tau(s)=m(s) \ddot{s}+c(s) \dot{s}^{2}+g(s),
$$

where

$$
\begin{aligned}
m(s) & =M(q(s)) q^{\prime}(s), \\
c(s) & =M(q(s)) q^{\prime \prime}(s)+C\left(q(s), q^{\prime}(s)\right) q^{\prime}(s) .
\end{aligned}
$$

The time-optimal path tracking problem for the manipulator can then be expressed as

$$
\begin{array}{cl}
\underset{s(\cdot), \tau(\cdot), T}{\operatorname{minimize}} & T \\
\text { subject to } & \tau(s(t))=m(s(t)) \ddot{s}(t)+c(s(t)) \dot{s}(t)^{2}+g(s(t)), \\
& s(0)=0, \\
& s(T)=1, \\
& \dot{s}(0)=\dot{s_{0}}, \\
& \dot{s}(T)=\dot{s}_{T}, \\
& \dot{s}(t) \geq 0, \\
& \underline{\tau}(s(t)) \leq \tau(s(t)) \leq \bar{\tau}(s(t)), \\
& \forall t \in[0, T]
\end{array}
$$

where $\underline{\tau}$ and $\bar{\tau}$ are the lower and upper bounds on the torques, respectively, and can be a function of $s$, and where $\dot{s}_{0}$ and $\dot{s}_{T}$ are, respectively, the initial and final velocities along the path, usually chosen to be 0 . In the above optimization problem, the inequalities are interpreted as component-wise inequalities.

\subsubsection{Convex formulation}

As shown in Verscheure et al. [2009], by introducing

$$
\begin{aligned}
& a(s)=\ddot{s}(t), \\
& b(s)=\dot{s}(t)^{2},
\end{aligned}
$$

as optimization variables, together with the additional constraint

$$
b^{\prime}(s)=2 a(s),
$$

and by a change of integration variable from $t$ to $s$ in the objective function in (3.4), the problem (3.4) can be reformulated as a convex optimization problem in which time $t$ does not appear anymore and $s$ acts as a pseudo-time. The constraint (3.6) follows from comparing the relations

$$
\begin{aligned}
& \dot{b}(s(t))=b^{\prime}(s) \dot{s}(t), \\
& \dot{b}(s(t))=\frac{d\left(\dot{s}(t)^{2}\right)}{d t}=2 \ddot{s}(t) \dot{s}(t)=2 a(s) \dot{s}(t),
\end{aligned}
$$


and the change of integration variable from $t$ to $s$ allows to write the duration of the motion as

$$
T=\int_{0}^{T} 1 d t=\int_{s(0)}^{s(T)} \frac{1}{\dot{s}(t)} d s=\int_{0}^{1} \frac{1}{\sqrt{b(s)}} d s .
$$

It must be noted that the nonlinear change of variables (3.5) is already recognized in several works, for example, in Pfeiffer and Johanni [1987] where it is used for geometric characterization of the admissible area of the motion, but using these transformed variables directly as optimization variables is proposed by Verscheure et al. [2009]. The resulting convex optimization problem from this change of variables can be written as

$$
\begin{array}{cl}
\underset{a(\cdot), b(\cdot), \tau(\cdot)}{\operatorname{minimize}} & \int_{0}^{1} \frac{1}{\sqrt{b(s)}} d s \\
\text { subject to } & \tau(s)=m(s) a(s)+c(s) b(s)+g(s), \\
& b(0)=\dot{s}_{0}^{2}, \\
& b(1)=\dot{s}_{T}^{2}, \\
& b(s) \geq 0, \\
& b^{\prime}(s)=2 a(s), \\
& \underline{\tau}(s) \leq \tau(s) \leq \bar{\tau}(s), \\
& \forall s \in[0,1] .
\end{array}
$$

This optimization problem is convex since the objective function is convex in $b(s)$, which follows from the fact that the integration preserves convexity and that $1 / \sqrt{b(s)}$ is convex in $b(s)$ (Boyd et al. [2004]), and since all the constraints are linear in the optimization variables. Extensions of the problem to incorporate other objective functions and constraints that preserve convexity can be found in Verscheure et al. [2009]. In particular, it is shown that symmetric lower and upper bounds on the joint velocities can be translated into upper bounds on $b(s)$. We denote this upper bound by $\bar{b}(s)$, and the corresponding constraint will be incorporated into the forthcoming forms of the problem (3.9).

The convex optimization problem (3.9) is an infinite dimensional problem with infinitely many optimization variables and constraints. Therefore, a finite parameterization of the optimization variables is employed to numerically solve the problem (3.9), which is presented in the following section.

\subsubsection{Numerical approach}

In order to numerically solve the optimization problem (3.9), the work by Verscheure et al. [2009] has proposed a direct transcription method based on the assumption that $a(s)=\frac{1}{2} b^{\prime}(s)$ is piecewise constant, since $a(s)$ can be seen as a control input. Based on this assumption, it follows that $b(s)$ and $\tau_{i}(s)$, where the 
subindex $i \in\{1, \ldots, n\}$ corresponds to the $i$ th joint, are piecewise linear and nonlinear, respectively. Using the direct transcription method, involves discretizing $s$ on $K+1$ grid points $s^{0}=0 \leq s^{k} \leq 1=s^{K}$ and modeling the functions $a(s), b(s)$ and $\tau_{i}(s)$ by, respectively, a finite number of variables $a^{k}, b^{k}$ and $\tau_{i}^{k}$, and results in a large sparse optimization problem. The variables $b^{k}$ are defined on the grid points $s^{k}$, i.e., $b^{k}=b\left(s^{k}\right)$, while $a^{k}$ and $\tau_{i}^{k}$ are defined in between the grid points, i.e., $a^{k}=a\left(s^{k+1 / 2}\right)$ and $\tau_{i}^{k}=\tau_{i}\left(s^{k+1 / 2}\right)$ where $s^{k+1 / 2}=\left(s^{k}+s^{k+1}\right) / 2$. Based on this direct transcription scheme, the problem (3.9) together with the velocity constraint can be written in discretized form as the following large scale optimization problem:

$$
\begin{array}{ll}
\underset{a^{k}, b^{k}, \tau^{k}}{\operatorname{minimize}} & \sum_{k=0}^{K-1} \frac{2 \Delta s^{k}}{\sqrt{b^{k+1}}+\sqrt{b^{k}}} \\
\text { subject to } & \tau^{k}=m\left(s^{k+1 / 2}\right) a^{k}+c\left(s^{k+1 / 2}\right) \frac{\left(b^{k}+b^{k+1}\right)}{2}+g\left(s^{k+1 / 2}\right), \\
& b^{0}=\dot{s}_{0}^{2}, \\
& b^{K}=\dot{s}_{T}^{2}, \\
& b^{k} \geq 0, b^{K} \geq 0, \\
& b^{k} \leq \bar{b}\left(s^{k}\right), b^{K} \leq \bar{b}\left(s^{K}\right), \\
& b^{k+1}-b^{k}=2 a^{k} \Delta s^{k}, \\
& \underline{\tau}\left(s^{k+1 / 2}\right) \leq \tau^{k} \leq \bar{\tau}\left(s^{k+1 / 2}\right), \\
& \text { for } k=0, \ldots, K-1,
\end{array}
$$

where the objective function is obtained by analytically calculating (3.8) as a sum of integrals over $\left[s^{k}, s^{k+1}\right]$ for $k=0, \ldots, K-1, \Delta s^{k}=s^{k+1}-s^{k}$, and $\tau^{k}=$ $\left[\tau_{1}^{k} \tau_{2}^{k} \ldots \tau_{n}^{k}\right]^{T}$. As demonstrated in Verscheure et al. [2009], this problem can be transformed into an SOCP, which can then be solved even more efficiently using the dedicated solvers for SOCPs. This transformation is presented in the next section.

\subsubsection{Second-order cone program formulation}

Let us consider the discretized problem (3.10). To obtain an SOCP, variables $d^{k}, k=0, \ldots, K-1$ are first introduced such that the minimization of the objective function can be equivalently written as

$$
\begin{array}{ll}
\operatorname{minimize} & \sum_{k=0}^{K-1} 2 \Delta s^{k} d^{k} \\
\text { subject to } & \frac{1}{\sqrt{b^{k+1}}+\sqrt{b^{k}}} \leq d^{k}, \quad k=0, \ldots, K-1 .
\end{array}
$$


The next step is to replace each constraint in (3.11) with two equivalent constraints by introducing variables $c^{k}, k=0, \ldots, K$ in the following way:

$$
\begin{aligned}
\frac{1}{c^{k+1}+c^{k}} & \leq d^{k}, \quad k=0, \ldots, K-1, \\
c^{k} & \leq \sqrt{b^{k}}, \quad k=0, \ldots, K,
\end{aligned}
$$

which in turn can be written as the following second-order cone constraints:

$$
\begin{gathered}
\left\|\begin{array}{c}
2 \\
c^{k}+c^{k+1}-d^{k}
\end{array}\right\|_{2} \leq c^{k}+c^{k+1}+d^{k}, \quad k=0, \ldots, K-1, \\
\left\|\begin{array}{c}
2 c^{k} \\
b^{k}-1
\end{array}\right\|_{2} \leq b^{k}+1, \quad k=0, \ldots, K .
\end{gathered}
$$

Using the constraints (3.13) together with the objective function in (3.11), the following SOCP is obtained:

$$
\begin{aligned}
\underset{a^{k}, b^{k}, \tau^{k}, d^{k}, c^{k}}{\operatorname{minimize}} & \sum_{k=0}^{K-1} 2 \Delta s^{k} d^{k} \\
\text { subject to } & \tau^{k}=m\left(s^{k+1 / 2}\right) a^{k}+c\left(s^{k+1 / 2}\right) \frac{\left(b^{k}+b^{k+1}\right)}{2}+g\left(s^{k+1 / 2}\right), \\
& b^{0}=\dot{s}_{0}^{2} \\
& b^{K}=\dot{s}_{T}^{2}, \\
& b^{k+1}-b^{k}=2 a^{k} \Delta s^{k}, \\
& { }^{\tau}\left(s^{k+1 / 2}\right) \leq \tau^{k} \leq \bar{\tau}\left(s^{k+1 / 2}\right), \\
& \left\|c^{k}+c^{k+1}-d^{k}\right\|_{2} \leq c^{k}+c^{k+1}+d^{k} \\
& \text { for } k=0, \ldots, K-1, \\
& b^{k} \geq 0, \\
& b^{k} \leq \bar{b}\left(s^{k}\right), \\
& \left\|\begin{array}{l}
2 c^{k} \\
b^{k}-1
\end{array}\right\|_{2} \leq b^{k}+1, \\
& \text { for } k=0, \ldots, K,
\end{aligned}
$$

where the variables $a^{k}, \tau^{k}, d^{k}$ are defined for $k=0, \ldots, K-1$, and $b^{k}, c^{k}$ for $k=$ $0, \ldots, K$.

The path tracking problems dealt with in this thesis are implemented and solved in SOCP form in MATLAB using the optimization modeling toolbox YALMIP (Lofberg [2004]). For solving the problems, we have used the solver MOSEK (ApS [2021]). 



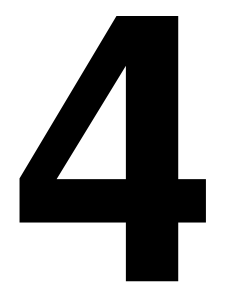

\section{Preliminary developments}

This chapter presents a preliminary study of the time-optimal cooperative path tracking problem. We show how the results in Verscheure et al. [2009] can be extended to solve the time-optimal path tracking problem for a particular setup where two manipulators and an object are involved. First, we consider a simplified scenario where there is no interaction between the manipulators. Second, we study the scenario where the two manipulators are holding an object with the objective of moving it along a predefined geometric path in minimum time. We show that the corresponding optimization problem can be cast as a convex problem.

An introduction to time-optimal path tracking for cooperative manipulators is given in the next section. Section 4.2 states the problem of interest in this chapter. Sections 4.3 and 4.4 extend the results in Chapter 3 to the first and second scenarios mentioned above, respectively. Finally, some simulation results are presented in Section 4.5.

\subsection{Introduction}

Research on time-optimal path tracking for cooperative manipulators has to take account of some issues that may not arise in the time-optimal path tracking problem for a single manipulator. Issues such as the incorporation of the object dynamics (Chang et al. [2000]; Erhart and Hirche [2016]), the imposed kinematic constraints on the emerging system (Erhart and Hirche [2013]), the distribution of the load among the manipulators (Tsiamis et al. [2015]), and the role of the internal forces (Erhart and Hirche [2015]; Verginis et al. [2019]), to name a few. A load distribution strategy in cooperative manipulation tasks allocates suitable torque and force setpoints to the manipulators in order to perform a desired action on the manipulated object. In this chapter we consider an equal load distri- 
bution for the manipulators. Other issues such as the incorporation of the object dynamics are discussed to some extent in this chapter, but will be addressed in more general form in the next chapters. In particular, a load distribution free of internal forces is presented in Chapter 6.

Related to the time-optimal path tracking, the work by Hollerbach [1983] proposes the time scaling of trajectories where a scaling factor is introduced to guarantee the feasibility of a planned trajectory. This approach is extended for cooperative robots in the work by Moon and Ahmad [1991], where it is shown that linear programming can be used to find the scaling factor that minimizes the traversal time for a give velocity profile. Time-optimal path tracking for cooperative multi-manipulator systems was independently studied by Bobrow et al. [1990] and Moon and Ahmad [1990], where linear programming was used to find an admissible phase plane boundary. This admissible phase plane region, similar to the approach in Bobrow et al. [1985] for a single manipulator, was employed to develop algorithms that construct the optimal trajectory. The work by Chen [1990] studies the problem of the existence and the structure of the minimumtime control for multiple manipulators cooperatively handling an object. It is shown that the minimum-time control requires that at least one of the actuators is always saturated on any finite time subinterval.

\subsection{Problem statement}

This section describes the time-optimal path tracking problem considered in this chapter. Consider two planar manipulators with two actuated joints and a nonactuated end-effector, rigidly grasping a bar as in Figure 4.1. The objective is to cooperatively move the bar along a prescribed geometric path in minimum time and subject to constraints on the joint torques, while keeping the bar perpendicular to the path. In this setup, the dynamics of the $i$ th manipulator is given by (2.8).

For now, we defer our discussion of this problem to Section 4.4, and start with a simplified scenario in the next section.

\subsection{Simplified scenario}

Here, we aim to extend the results in Section 3.2 to the case where there are two manipulators, but still keep the problem as simple as possible.

We consider the following scenario; two manipulators with a common path to follow but without interacting through a common load. In this scenario, the end-effectors of the two robots are supposed to be at the same point on the path at each time instant. For simplicity, we assume that there is no interaction between the two end-effectors and the force exchanged between them is zero. Although this scenario is not realistic, it serves as a useful introduction to the problem of interest in this chapter.

Assume that the path is expressed in the Cartesian space as a function of the path coordinate $s$ and with respect to a world frame, and assume that each ma- 


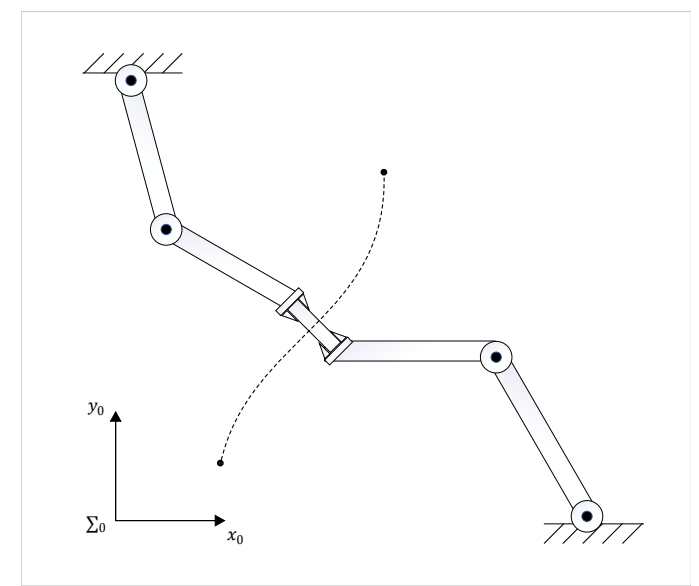

Figure 4.1: Schematic representation of the manipulators and the bar.

nipulator has its own reference frame. For a path given in the operational space coordinates, inverse kinematics can be employed to compute the corresponding path in the joint space coordinates (Slotine and Yang [1988]). Hence, a joint space representation of the path can be obtained for each manipulator, which we denote by $q_{1}(s)$ and $q_{2}(s)$. Note that, since the end-effectors are at the same position at each time instant and follow the same path, the mapping between the time and path coordinate can be chosen to be the same for both manipulators. This allows us to express $q_{1}(\cdot)$ and $q_{2}(\cdot)$ as functions of a common path coordinate $s(t)$. Consequently, the robots will have the same variables $a(s)$ and $b(s)$ defined in (3.5). Hence, we can employ them as the common optimization variables for both manipulators in the corresponding optimization problem.

Based on the discussion above and the procedure employed in Section 3.2, the problem of interest in this section can be cast as the convex optimization problem (3.9) with the difference that, there will be more constraints accounting for the second manipulator's dynamics and torque bounds, i.e., the constraints (3.9b) and $(3.9 \mathrm{~g})$ are replaced by

$$
\tau_{i}(s)=m_{i}(s) a(s)+c_{i}(s) b(s)+g_{i}(s), \quad i=1,2
$$

and

$$
\underline{\tau}_{i}(s) \leq \tau_{i}(s) \leq \bar{\tau}_{i}(s), \quad i=1,2,
$$

respectively, where the subindex $i$ refers to the $i$ th manipulator. It should be noted that the joint torques of the second manipulator, i.e., $\tau_{2}(s)$, will also be included in the optimization variables.

Similar to the procedure employed in Verscheure et al. [2009], this problem can finally be formulated as an SOCP. 


\subsection{Time-optimal cooperative path tracking}

We are now ready to present the main result of the chapter. Consider the problem described in Section 4.2. To solve this problem, the forces acting on the bar are computed and split into two parts, and then merged with the corresponding manipulator's dynamics. In this way we get two sets of equations, describing the motion of the whole system, which makes it possible to use the results obtained for two manipulators in the previous section.

Before going into the details, let us introduce some notation. Given a vector $x$, let $\|x\|$ be its Euclidean norm. The quantity $\hat{k}$ is the unit vector in the direction of the $z$-axis. The world frame is denoted by $\Sigma_{0}$. Let $p(s)=\left[p_{1}(s) p_{2}(s)\right]^{T} \in \mathbb{R}^{2}$ be the desired path of the bar's center of mass, given in Cartesian space with respect to $\Sigma_{0}$. Let $m_{b}$ denote the mass of the bar. We assume that the bar's center of mass is at the same distance from both ends of the bar, and we denote this distance by $d$. We denote by $q_{i 1}$ and $q_{i 2}$ the first and second components of the joint angle $q_{i}(s)$, respectively. Lengths of the two links of the $i$ th manipulator are denoted by $l_{i 1}$ and $l_{i 2}$, respectively.

We begin by deriving the forces acting on the bar. These forces can be decomposed into two components, the translational and the rotational forces, denoted by $F_{t}$ and $F_{r}$, respectively. The translational part is obtained by $F_{t}(s)=m_{b} \ddot{p}(s)$, where $\ddot{p}(s)$ is the acceleration along the path. Using the chain rule, the acceleration can be rewritten as $\ddot{p}(s)=p^{\prime}(s) \ddot{s}+p^{\prime \prime}(s) \dot{s}^{2}$. Substituting this into the above equation for $F_{t}$, yields the following expression for the translational part:

$$
F_{t}(s)=m_{b} p^{\prime}(s) \ddot{s}+m_{b} p^{\prime \prime}(s) \dot{s}^{2} .
$$

This force has the same effect on the bar as if there were two forces applied to both ends of the bar, in a direction same as $F_{t}(s)$, and a magnitude equal to half of the magnitude of $F_{t}(s)$, see Figure 4.2 for an illustration. Hence in our analysis, we can replace $F_{t}(s)$ with these two forces, which allows us to describe the dynamics of the whole system using two sets of equations, each representing the motion of a manipulator while taking account of the resulting forces from the bar's motion. Moreover, there is a gravity force acting on the bar, denoted $F_{g}$, which can be treated in a similar way to $F_{t}(s)$.

In order to compute the rotational component, first, we consider the torque applied to the bar, which is denoted by $\tau_{b}$. Let $I$ denote the inertia matrix of the bar with respect to a body-fixed frame whose origin is at the center of mass and its $x$-axis is aligned along the bar, and let $R$ be the rotation matrix that transforms coordinates from the body-fixed frame to $\Sigma_{0}$. Then the torque applied to the bar is easily computed by [Spong et al., 2006],

$$
\tau_{b}(s)=\frac{d}{d t}\left(R(s) I R(s)^{T} \omega(s)\right),
$$

where $\omega(s)$ is the angular velocity of the bar. Let $\phi$ be the angle between the bar and the $x$-axis of the inertial frame. When the configuration is as in Figure 4.1, since $\omega(s)$ is aligned with the $z$-axis and $R(s)$ is a rotation matrix about the $z$-axis, 


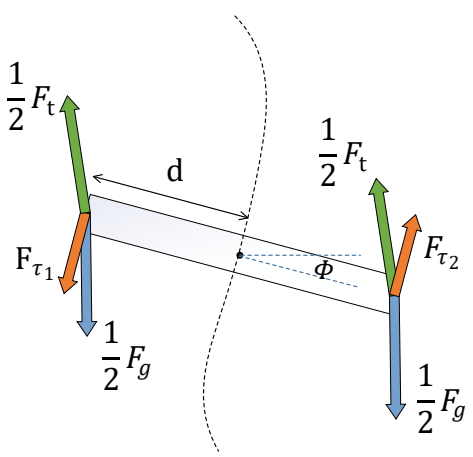

Figure 4.2: Schematic representation of the forces acting on the bar.

(4.4) simply reduces to

$$
\tau_{b}(s)=I_{z z} \ddot{\phi}(s) \hat{k}
$$

by replacing $\omega(s)$ with $\dot{\phi}(s) \hat{k}$, where $I_{z z}$ is the moment of inertia about the $z$-axis of the bar's body-fixed frame. Since the bar is perpendicular to the path at each $s$, the angle $\phi(s)$ can be obtained by $\phi(s)=\arctan \left(-p_{2}^{\prime}(s) / p_{1}^{\prime}(s)\right)$, where $p_{1}^{\prime}(s)$ and $p_{2}^{\prime}(s)$ are the first and second components of $p^{\prime}(s)$, respectively.

The torque (4.5) can be viewed as the result of two forces $F_{\tau_{1}}$ and $F_{\tau_{2}}$, acting on both ends of the bar at a right angle, with the same magnitude but in opposite directions, as shown in Figure 4.2. Observing that these forces are parallel to $p^{\prime}(s)$, which is a vector tangent to the path, and the fact that their magnitudes and the magnitude of $\tau_{b}$ are related by $2 d\left\|F_{\tau_{i}}(s)\right\|=\left\|\tau_{b}(s)\right\|, i=1,2$, it follows that,

$$
F_{\tau_{1}}(s)=\frac{-I_{z z} \ddot{\phi}(s) p^{\prime}(s)}{2 d\left\|p^{\prime}(s)\right\|},
$$

and $F_{\tau_{2}}(s)=-F_{\tau_{1}}(s)$. Additionally, using

$$
\ddot{\phi}(s)=\phi^{\prime}(s) \ddot{s}+\phi^{\prime \prime}(s) \dot{s}^{2}
$$

in equation (4.6) we have,

$$
F_{\tau_{1}}(s)=\left(\frac{-I_{z z} \phi^{\prime}(s) p^{\prime}(s)}{2 d\left\|p^{\prime}(s)\right\|}\right) \ddot{s}+\left(\frac{-I_{z z} \phi^{\prime \prime}(s) p^{\prime}(s)}{2 d\left\|p^{\prime}(s)\right\|}\right) \dot{s}^{2}
$$

Now, the net force acting on each end of the bar can be obtained by adding the different forces computed above. Let $F_{i}^{0}$ denote the net force exerted on the 
end located at the $i$ th manipulator side. Then $F_{i}^{0}$ becomes,

$$
F_{i}^{0}(s)=\frac{1}{2} F_{t}(s)+F_{\tau_{i}}(s)+\frac{1}{2} F_{g}
$$

which, using (4.3) and (4.7), can be written as,

$$
F_{i}^{0}(s)=\bar{m}_{i}(s) \ddot{s}+\bar{c}_{i}(s) \dot{s}^{2}+\bar{g},
$$

where,

$$
\begin{aligned}
\bar{m}_{1}(s) & =\frac{1}{2} m_{b} p^{\prime}(s)-\frac{I_{z z} \phi^{\prime}(s) p^{\prime}(s)}{2 d\left\|p^{\prime}(s)\right\|}, \\
\bar{c}_{1}(s) & =\frac{1}{2} m_{b} p^{\prime \prime}(s)-\frac{I_{z z} \phi^{\prime \prime}(s) p^{\prime}(s)}{2 d\left\|p^{\prime}(s)\right\|}, \\
\bar{g} & =\left[\begin{array}{c}
0 \\
-\frac{1}{2} m_{b} a_{g}
\end{array}\right], \\
\bar{m}_{2}(s) & =\frac{1}{2} m_{b} p^{\prime}(s)+\frac{I_{z z} \phi^{\prime}(s) p^{\prime}(s)}{2 d\left\|p^{\prime}(s)\right\|}, \\
\bar{c}_{2}(s) & =\frac{1}{2} m_{b} p^{\prime \prime}(s)+\frac{I_{z z} \phi^{\prime \prime}(s) p^{\prime}(s)}{2 d\left\|p^{\prime}(s)\right\|} .
\end{aligned}
$$

In the above expression for $\bar{g}, a_{g}$ is the gravitational acceleration.

The next step is to transform the net force $F_{i}^{0}(s)$, to an equivalent quantity in the $i$ th manipulator frame. The vector of forces and moments exerted by the $i$ th end-effector on the corresponding grasping point, and represented by $F_{i}=\left[F_{i x}, F_{i y}, F_{i z}, n_{i x}, n_{i y}, n_{i z}\right]^{T}$, requires torques at the joints of the corresponding manipulator, which we denote by $\bar{\tau}_{i}$. These two quantities are related by the use of the manipulator Jacobian (Spong et al. [2006]),

$$
\bar{\tau}_{i}(s)=-J_{i}\left(q_{i}(s)\right)^{T} F_{i}(s), \quad i=1,2,
$$

where $J_{i}\left(q_{i}(s)\right)$ is the $i$ th manipulator Jacobian. Note that in order to derive the joint angles $q_{i}(s)$, inverse kinematics should be applied to the path where the $i$ th end-effector is located, which is different from $p(s)$. We come back to this point later in our discussion.

With the forces decomposed as in Figure 4.2, there are no moments acting on the ends of the bar, and the forces are present either in the $x$-direction or $y$ direction, i.e., $F_{i}=\left[F_{i x}, F_{i y}, 0,0,0,0\right]^{T}$. In order to obtain $F_{i x}$ and $F_{i y}$, which are the components of the local representation of $F_{i}^{0}(s)$, we will make use of rotation matrices. For $i=1,2$, let $R_{0}^{i} \in \mathbb{R}^{2 \times 2}$ be the rotation matrix that specifies the orientation of the frame $\Sigma_{0}$ with respect to the $i$ th manipulator's local frame. Then

$$
\left[\begin{array}{l}
F_{i x}(s) \\
F_{i y}(s)
\end{array}\right]=R_{0}^{i} F_{i}^{0}(s), \quad i=1,2 .
$$


For the $i$ th planar manipulator with two links, the Jacobian can be obtained as, see Spong et al. [2006],

$$
J_{i}\left(q_{i}(s)\right)=\left[\begin{array}{c}
\bar{J}_{i}\left(q_{i}(s)\right) \\
w
\end{array}\right]
$$

where,

$$
\begin{aligned}
\bar{J}_{i}\left(q_{i}(s)\right) & =\left[\begin{array}{cc}
-l_{i 1} \sin \left(q_{i 1}\right)-l_{i 2} \sin \left(q_{i 1}+q_{i 2}\right) & -l_{i 2} \sin \left(q_{i 1}+q_{i 2}\right) \\
l_{i 1} \cos \left(q_{i 1}\right)+l_{i 2} \cos \left(q_{i 1}+q_{i 2}\right) & l_{i 2} \cos \left(q_{i 1}+q_{i 2}\right)
\end{array}\right], \\
w & =\left[\begin{array}{llll}
0 & 0 & 0 & 1 \\
0 & 0 & 0 & 1
\end{array}\right]^{T} .
\end{aligned}
$$

Using (4.11) and (4.12) in (4.10), one has

$$
\bar{\tau}_{i}(s)=-\bar{J}_{i}\left(q_{i}(s)\right)^{T} R_{0}^{i} F_{i}^{0}(s), \quad i=1,2 .
$$

Then, using (4.9) in (4.15), $\bar{\tau}_{i}(s)$ similar to (4.1), can be expressed as a function of $\ddot{s}, \dot{s}^{2}$ and $s$, as

$$
\bar{\tau}_{i}(s)=-\left(\bar{J}_{i}\left(q_{i}(s)\right)^{T} R_{0}^{i} \bar{m}_{i}(s)\right) \ddot{s}-\left(\bar{J}_{i}\left(q_{i}(s)\right)^{T} R_{0}^{i} \bar{c}_{i}(s)\right) \dot{s}^{2}-\left(\bar{J}_{i}\left(q_{i}(s)\right)^{T} R_{0}^{i} \bar{g}\right) .
$$

Finally, adding (4.16) to $\tau_{i}(s)$ in (4.1) gives rise to two sets of equations which determine the torques required to achieve the desired motion of the overall system, including the bar. That is, for $i=1,2$,

$$
\begin{aligned}
\tau_{i}(s)= & \left(m_{i}(s)+\bar{J}_{i}\left(q_{i}(s)\right)^{T} R_{0}^{i} \bar{m}_{i}(s)\right) a(s)+\left(c_{i}(s)+\bar{J}_{i}\left(q_{i}(s)\right)^{T} R_{0}^{i} \bar{c}_{i}(s)\right) b(s) \\
& +\left(g_{i}(s)+\bar{J}_{i}\left(q_{i}(s)\right)^{T} R_{0}^{i} \bar{g}\right) .
\end{aligned}
$$

Another issue to be discussed is how to obtain $q_{i}(s)$ from the given path for the bar's center of mass, $p(s)$. To this end, first, the two paths where the end-effectors are located should be determined. This can be done by observing that the bar is always perpendicular to $p(s)$, and that the distances between its center of mass and each of the paths of interest are $d$. This leads us to the following paths,

$$
\begin{aligned}
& \bar{p}_{1}(s)=\left[\begin{array}{l}
p_{1}(s)-\frac{d}{\left\|p^{\prime}(s)\right\|} p_{2}^{\prime}(s) \\
p_{2}(s)+\frac{d}{\left\|p^{\prime}(s)\right\|} p_{1}^{\prime}(s)
\end{array}\right], \\
& \bar{p}_{2}(s)=\left[\begin{array}{l}
p_{1}(s)+\frac{d}{\left\|p^{\prime}(s)\right\|} p_{2}^{\prime}(s) \\
p_{2}(s)-\frac{d}{\left\|p^{\prime}(s)\right\|} p_{1}^{\prime}(s)
\end{array}\right] .
\end{aligned}
$$

Each one of these paths should be assigned to one of the manipulators, and this is determined by the configuration of the robots and how they are located with respect to the given path for the bar's center of mass. Then one can use inverse kinematics techniques to obtain the corresponding $q_{i}(s)$ for each robot. 
Now, with representation (4.17) for the dynamics of the manipulators together with the motion of the bar, it is possible to cast the problem of interest as the following convex optimization problem:

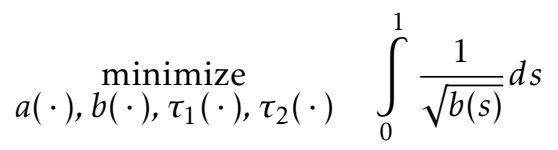

$$
\begin{aligned}
& \text { subject to } \quad \tau_{i}(s)=\left(m_{i}(s)+\bar{J}_{i}\left(q_{i}(s)\right)^{T} R_{0}^{i} \bar{m}_{i}(s)\right) a(s) \\
& +\left(c_{i}(s)+\bar{J}_{i}\left(q_{i}(s)\right)^{T} R_{0}^{i} \bar{c}_{i}(s)\right) b(s) \\
& +\left(g_{i}(s)+\bar{J}_{i}\left(q_{i}(s)\right)^{T} R_{0}^{i} \bar{g}\right), \quad i=1,2, \\
& b(0)=\dot{s}_{0}^{2} \text {, } \\
& b(1)=\dot{s}_{T}^{2} \text {, } \\
& b(s) \geq 0 \text {, } \\
& b(s) \leq \bar{b}(s) \text {, } \\
& b^{\prime}(s)=2 a(s) \text {, } \\
& \underline{\tau}_{i}(s) \leq \tau_{i}(s) \leq \bar{\tau}_{i}(s), \quad i=1,2 \text {, } \\
& \forall s \in[0,1] \text {. }
\end{aligned}
$$

It is worth noticing that the resulting equation for the joint torques from the transformation of the forces related to the bar, has the same form as (4.1), which plays a key role in our analysis. The problem (4.20) can also be reformulated as an SOCP after employing the direct transcription method.

\subsection{Numerical simulation}

In this section, we include some numerical simulations to illustrate the results. Consider the problem setup presented in Section 4.2. The mass of the bar and the parameter $d$ are considered to be $1 \mathrm{~kg}$ and $0.15 \mathrm{~m}$, respectively. Table 4.1 provides the dynamic parameters used in the simulation for both manipulators, where $m_{i j}$ and $l_{i j}$ denote the mass and the length of the $j$ th link of the $i$ th manipulator, respectively.

Table 4.1: Dynamic parameters

\begin{tabular}{|l|cc|cc|}
\hline & $m_{i 1}(\mathrm{~kg})$ & $m_{i 2}(\mathrm{~kg})$ & $l_{i 1}(\mathrm{~m})$ & $l_{i 2}(\mathrm{~m})$ \\
\hline First manipulator $(i=1)$ & 6.1 & 4.4 & 1.6 & 1.2 \\
\hline Second manipulator $(i=2)$ & 6 & 5 & 1.5 & 1.3 \\
\hline
\end{tabular}

The desired path is given in the XY-plane by,

$$
p(s)=\left[\begin{array}{c}
2.2 s+0.58 \\
7.15(s-0.5)^{3}+1.65
\end{array}\right]
$$




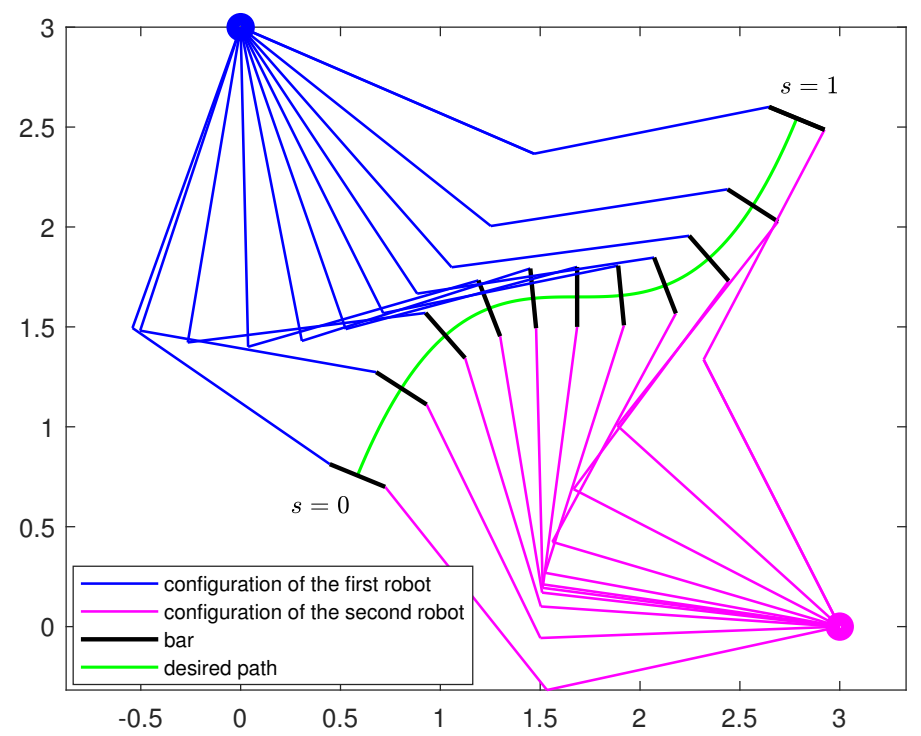

Figure 4.3: Desired path and configurations of the manipulators and the bar.

Figure 4.3 illustrates this path as well as the configurations of the manipulators and the bar in steps of 0.1 .

The optimization problem is formulated as an SOCP and is solved using YALMIP Lofberg [2004] and MOSEK ApS [2021] toolboxes in MATLAB.

The resulting joint torques and the corresponding bounds, for the first and second manipulators are shown as a function of path coordinate $s$ in Figure 4.4 and Figure 4.5, respectively. From the plots, it can be seen that for every point along the path, one of the joint torques of either the first or the second manipulator is in saturation, which is consistent with the results that can be found in the literature on minimum-time control of a single manipulator, see e.g., Chen and Desrochers [1989].

To compute the joint velocities and accelerations, once $a(s)$ and $b(s)$ are obtained, we can use (3.1a) and (3.1b), respectively. Figure 4.6 and Figure 4.7 illustrate the evolution of the joint velocities with respect to the path coordinate $s$ for the first and second robots, respectively. The initial and final velocities are chosen to be zero by setting $\dot{s}_{0}$ and $\dot{s}_{T}$ to zero, which is the case in most applications. 


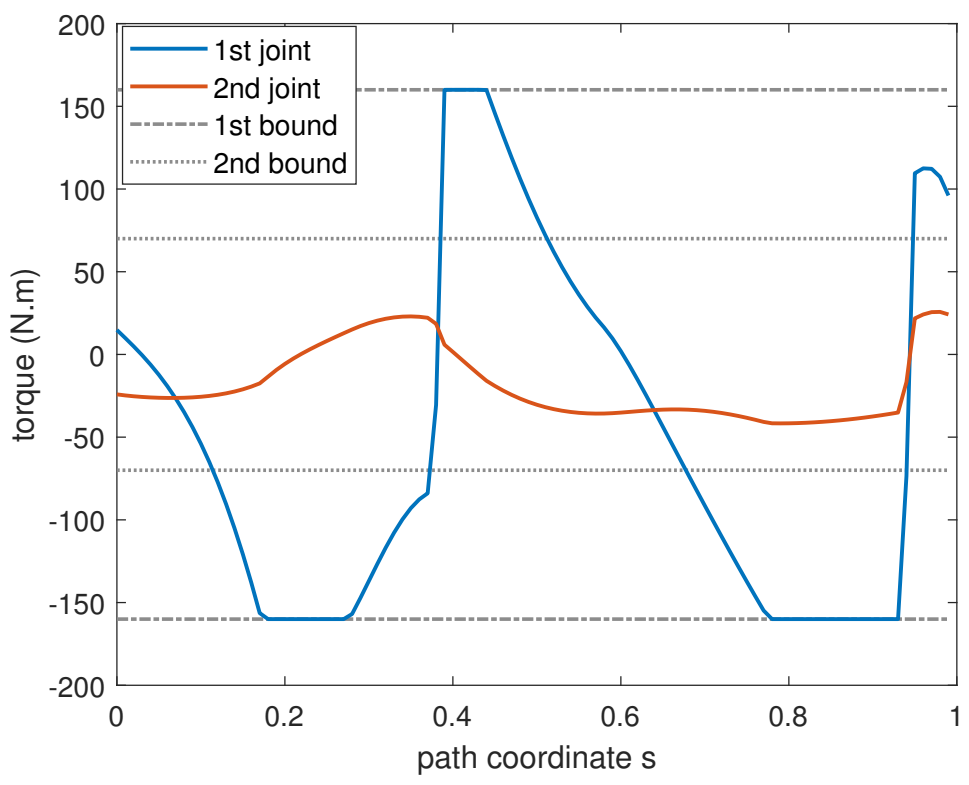

Figure 4.4: Joint torques $\tau_{1}(s)$

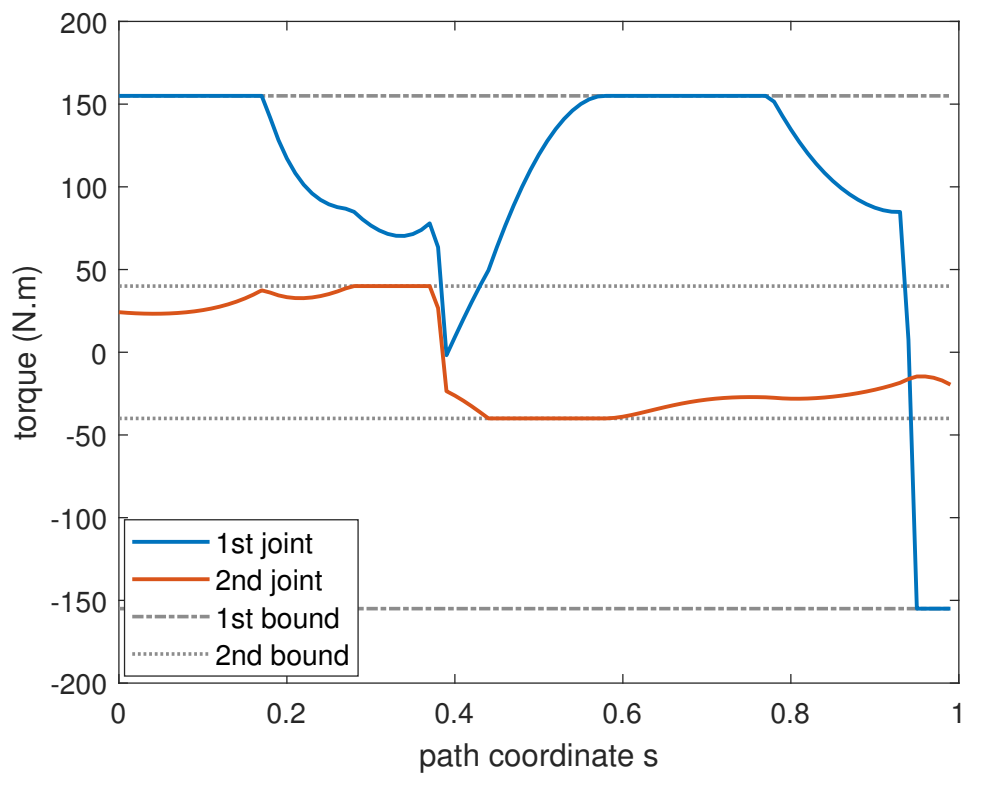

Figure 4.5: Joint torques $\tau_{2}(s)$ 


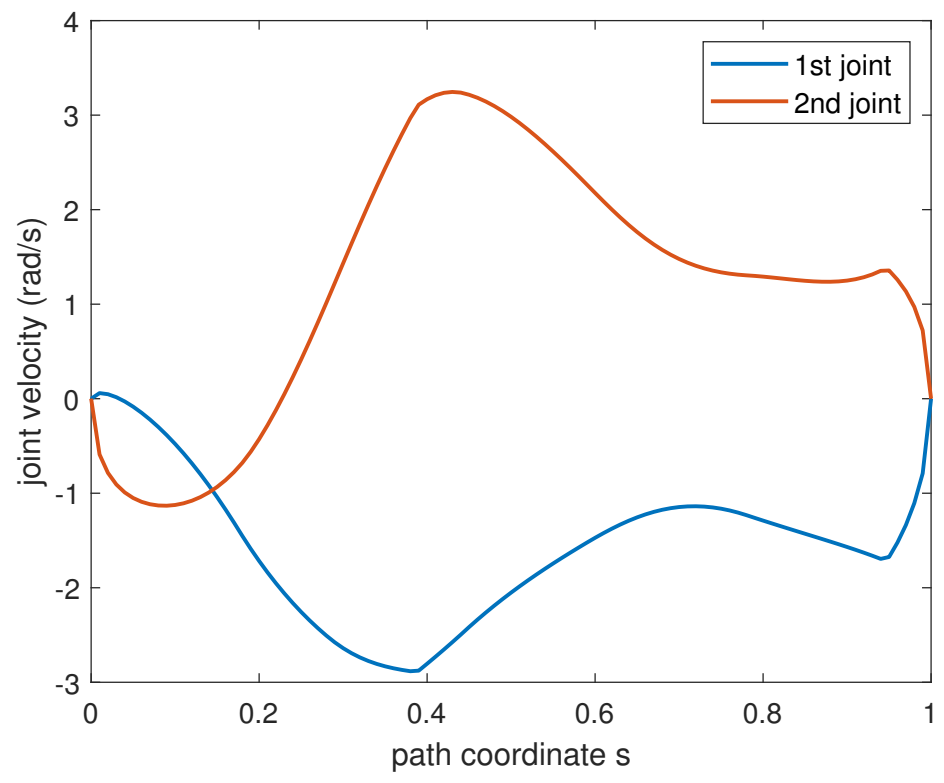

Figure 4.6: Joint velocities $\dot{q}_{1}(s)$

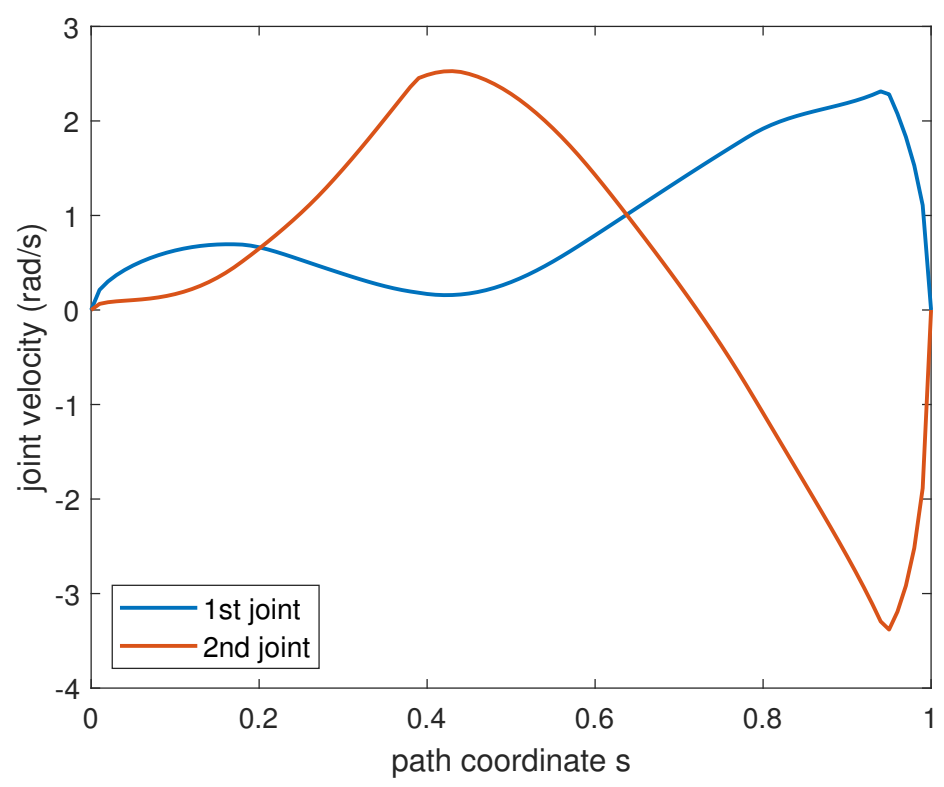

Figure 4.7: Joint velocities $\dot{q}_{2}(s)$ 



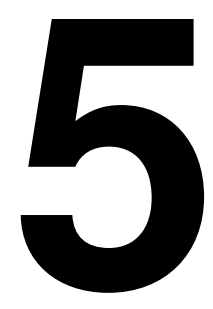

\section{General framework for time-optimal cooperative path tacking}

This chapter addresses the problem of time-optimal path tracking for multiple cooperative manipulators rigidly grasping an object. In the previous chapter, a preliminary study of this problem was presented for a particular setup with two planar manipulators. The aim of this chapter is to generalize those results to a more general scenario with $N$ manipulators and an object with a desired orientation during the motion. In Chapter 4 , an equal load distribution was considered for the manipulators, while in this chapter the load distribution is determined via a pseudo-inverse of the grasp matrix that can be chosen by the user. The freedom in the choice of the pseudo-inverse allows to consider different load distributions which can be exploited to account for the potential differences in the capabilities of the manipulators.

The first section of the chapter introduces the problem of interest. Section 5.2 contains the main results of the chapter. Section 5.3 illustrate the results in simulation.

\subsection{Problem statement}

Consider $N$ fully actuated robotic manipulators, indexed by the set $\mathcal{N}=\{1, \ldots, N\}$, rigidly grasping an object as in Figure 5.1. The rigidity assumption implies that the manipulators can exert forces and moments along all directions to the object. Similar to the problem in Section 4.2, the objective here is to minimize the traversal time required to move the object with a desired orientation along a prescribed geometric path, where the path is given for the centre of mass of the object. The motion is subject to constraints on the joint torques and velocities. We assume that the prescribed geometric path and the object's orientation are given as functions of the scalar path coordinate $s$. Furthermore, as in Section 3.2.2, we assume that the trajectory starts at $t=0$, ends at $t=T, s(0)=0 \leq s(t) \leq s(T)=1$, and 


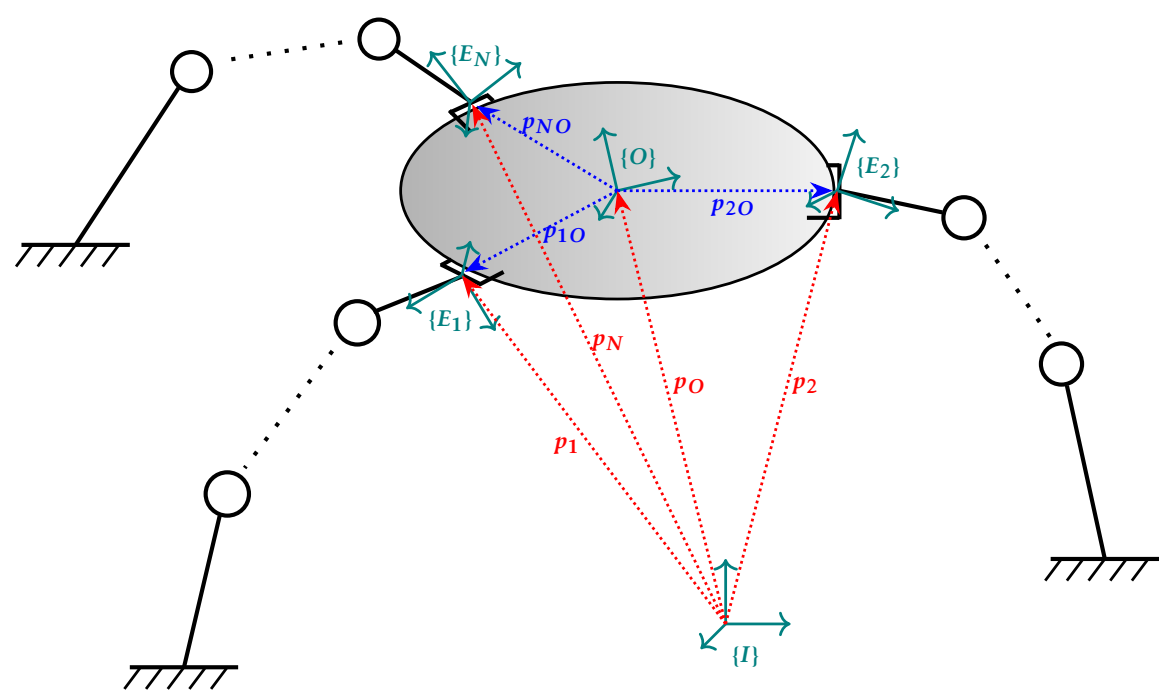

Figure 5.1: Three robotic manipulators rigidly grasping an object.

that $\dot{s}(t) \geq 0, \forall t \in[0, T]$.

Throughout the chapter, all quantities are expressed with respect to an inertial frame of reference $\{I\}$ whenever the reference frame is not explicitly indicated by a superscript. Let $\{O\}$ and $\left\{E_{i}\right\}$ denote the reference frames attached to the object at the centre of mass and the $i$ th end-effector, respectively. Let $\phi_{O}=\left[\varphi_{O}, \vartheta_{O}, \psi_{O}\right]^{T}$ be the object's orientation in terms of a triplet of Euler angles. If one is not concerned with the orientation of the object during the motion and only the initial and the final orientations are of interest, then $\phi_{O}$ can be chosen as

$$
\phi_{O}(s)=\phi_{O_{i}}+\left(\phi_{O_{f}}-\phi_{O_{i}}\right) s
$$

where $\phi_{O_{i}}$ and $\phi_{O_{f}}$ are the initial and the final orientations, respectively.

In the following, we present the modeling of the coupled kinematics and the dynamics of the manipulators and the object.

\subsubsection{Kinematics}

Let $p_{O} \in \mathbb{R}^{3}$ be the position of the object's centre of mass. Let $p_{i} \in \mathbb{R}^{3}$ and $\phi_{i}$ be the position and orientation of the $i$ th end-effector, respectively. In view of Figure 5.1, one can see that the pose of the $i$ th end-effector and the object's centre of mass are related by

$$
\begin{aligned}
& p_{i}(s)=p_{O}(s)+p_{i O}(s)=p_{O}(s)+R_{E_{i}}(s) p_{i O}^{E_{i}}, \\
& \phi_{i}(s)=\phi_{O}(s)+\phi_{i O},
\end{aligned}
$$

where $p_{i O}^{E_{i}}$ and $\phi_{i O}$ are the constant distance and orientation offsets between the reference frames $\{O\}$ and $\left\{E_{i}\right\}$, respectively, and are considered to be known. The 
$3 \times 3$ matrix $R_{E_{i}}$ denotes the rotation matrix of the reference frame $\left\{E_{i}\right\}$ with respect to $\{I\}$.

\subsubsection{Manipulator dynamics}

The dynamics of the $i$ th manipulator with $n_{i}$ DOF and the joint variables $q_{i} \in \mathbb{R}^{n_{i}}$ can be described by equation (2.9), where $h_{i} \in \mathbb{R}^{6}$ is the vector of generalized forces exerted by the $i$ th end-effector on the object.

Given $p_{O}(s)$ and $\phi_{O}(s)$, the pose of each end-effector can be computed by (5.2). Then, inverse kinematics can be employed to compute the corresponding joint variables as functions of the path coordinate $s$. Using the chain rule, the joint velocities and accelerations of the $i$ th manipulator can be written as

$$
\begin{aligned}
& \dot{q}_{i}(s)=q_{i}^{\prime}(s) \dot{s}, \\
& \ddot{q}_{i}(s)=q_{i}^{\prime}(s) \ddot{s}+q_{i}^{\prime \prime}(s) \dot{s}^{2},
\end{aligned}
$$

where $q_{i}^{\prime}(s)=\partial q_{i}(s) / \partial s, q_{i}^{\prime \prime}(s)=\partial^{2} q_{i}(s) / \partial s^{2}, \dot{s}=d s / d t$ and $\ddot{s}=d^{2} s / d t^{2}$. Substituting these expressions in (2.9) yields the following expression for the equations of motion:

$$
\tau_{i}(s)=m_{i}(s) \ddot{s}+c_{i}(s) \dot{s}^{2}+g_{i}(s)+J_{i}^{T}(s) h_{i},
$$

where

$$
\begin{aligned}
m_{i}(s) & =M_{i}\left(q_{i}(s)\right) q_{i}^{\prime}(s), \\
c_{i}(s) & =M_{i}\left(q_{i}(s)\right) q_{i}^{\prime \prime}(s)+C_{i}\left(q_{i}(s), q_{i}^{\prime}(s)\right) q_{i}^{\prime}(s),
\end{aligned}
$$

for each $i \in \mathcal{N}$.

\subsubsection{Object dynamics}

Regarding the object, the pose and the velocity of its centre of mass are denoted by $x_{O}=\left[p_{O}^{T}, \phi_{O}^{T}\right]^{T}$ and $v_{O}=\left[\dot{p}_{O}^{T}, \omega_{O}^{T}\right]^{T}$, respectively, where $\omega_{O} \in \mathbb{R}^{3}$ is the object's angular velocity. The following second-order dynamics, which is based on the Newton-Euler formulation can be considered for the object:

$$
M_{O}\left(x_{O}\right) \dot{v}_{O}+C_{O}\left(x_{O}, \dot{x}_{O}\right) v_{O}+g_{O}=h_{O},
$$

where

$$
\begin{aligned}
M_{O} & =\left[\begin{array}{cc}
m I_{3} & 0_{3 \times 3} \\
0_{3 \times 3} & I_{O}
\end{array}\right], \\
C_{O} & =\left[\begin{array}{cc}
0_{3 \times 3} & 0_{3 \times 3} \\
0_{3 \times 3} & S\left(\omega_{O}\right) I_{O}
\end{array}\right], \\
g_{O} & =\left[\begin{array}{c}
-m g \\
0_{3}
\end{array}\right],
\end{aligned}
$$

and where $m$ is the mass of the object, $g \in \mathbb{R}^{3}$ is the gravitational acceleration vector, $I_{O} \in \mathbb{R}^{3 \times 3}$ is the object's inertia tensor relative to the centre of mass when 
expressed in a frame parallel to $\{I\}$ with origin at the centre of mass, and $h_{O} \in \mathbb{R}^{6}$ is the vector of generalized forces acting on the object's centre of mass. Here, $S(\cdot)$ is the skew-symmetric operator. Given a vector $z=\left[z_{1}, z_{2}, z_{3}\right]^{T}$, the skewsymmetric matrix $S(z)$ is defined as

$$
S(z)=-S(z)^{T}=\left[\begin{array}{ccc}
0 & -z_{3} & z_{2} \\
z_{3} & 0 & -z_{1} \\
-z_{2} & z_{1} & 0
\end{array}\right]
$$

\subsection{Main results}

Now, we are ready to present the main result of the chapter. The first step is to show that the object dynamics (5.6), similar to equation (5.4) can be written as a function of $\ddot{s}, \dot{s}^{2}$ and $s$. This allows us to use $\ddot{s}$ and $\dot{s}^{2}$ as optimization variables later. To this end, we start by writing the first- and the second-order time derivatives of the pose of the object's centre of mass as

$$
\begin{aligned}
& \dot{x}_{O}(s)=x_{O}^{\prime}(s) \dot{s}, \\
& \ddot{x}_{O}(s)=x_{O}^{\prime}(s) \ddot{s}+x_{O}^{\prime \prime}(s) \dot{s}^{2},
\end{aligned}
$$

respectively, where $x_{O}^{\prime}(s)=\partial x_{O}(s) / \partial s$ and $x_{O}^{\prime \prime}(s)=\partial^{2} x_{O}(s) / \partial s^{2}$.

Next, we find the relationship between the velocity and the time derivative of the pose of the object's centre of mass. For this, we know that it is possible to find the relationship between the object's angular velocity $\omega_{O}$ and its rotational velocity $\dot{\phi}_{O}$ for a given set of orientation angles (Siciliano et al. [2010]). Let $T\left(\phi_{O}\right)$ be the transformation between these two velocities, i.e., $\omega_{O}=T\left(\phi_{O}\right) \dot{\phi}_{O}$. Using this equation, the velocity of the object's centre of mass can be written as

$$
v_{O}(s)=T_{O}(s) \dot{x}_{O}(s) \text {, }
$$

where

$$
T_{O}(s)=\left[\begin{array}{cc}
I_{3} & 0_{3 \times 3} \\
0_{3 \times 3} & T\left(\phi_{O}(s)\right)
\end{array}\right] .
$$

Substituting (5.9) into (5.10) and its derivative, yields the following expressions for $v_{O}$ and $\dot{v}_{O}$, respectively:

$$
\begin{aligned}
& v_{O}(s)=T_{O}(s) x_{O}^{\prime}(s) \dot{s}, \\
& \dot{v}_{O}(s)=\left(\frac{\partial T_{O}(s)}{\partial s} x_{O}^{\prime}(s)+T_{O}(s) x_{O}^{\prime \prime}(s)\right) \dot{s}^{2}+T_{O}(s) x_{O}^{\prime}(s) \ddot{s} .
\end{aligned}
$$

Finally, substituting (5.9) and (5.12) into (5.6), an expression for the object dynamics can be obtained as follows:

$$
m_{O}(s) \ddot{s}+c_{O}(s) \dot{s}^{2}+g_{O}=h_{O} \text {, }
$$


where

$$
\begin{aligned}
m_{O}(s)= & M_{O}\left(x_{O}(s)\right) T_{O}(s) x_{O}^{\prime}(s), \\
c_{O}(s)= & M_{O}\left(x_{O}(s)\right) \frac{\partial T_{O}(s)}{\partial s} x_{O}^{\prime}(s)+M_{O}\left(x_{O}(s)\right) T_{O}(s) x_{O}^{\prime \prime}(s) \\
& +C_{O}\left(x_{O}(s), x_{O}^{\prime}(s)\right) T_{O}(s) x_{O}^{\prime}(s) .
\end{aligned}
$$

The second step concerns the relationship between the generalized forces $h_{O}$ acting on the object's centre of mass and the generalized forces $h_{i}, i \in \mathcal{N}$ exerted by the end-effectors to the object at the grasping points.

Let $v_{i}=\left[\dot{p}_{i}^{T}, \omega_{i}^{T}\right]^{T}$ be the velocity of the $i$ th end-effector, where $\omega_{i} \in \mathbb{R}^{3}$ is the angular velocity. Differentiation of (5.2a) together with the fact that $\omega_{i}=\omega_{O}$ due to the grasping rigidity, leads to

$$
v_{i}(s)=J_{O_{i}}(s) v_{O}(s), \quad \forall i \in \mathcal{N},
$$

where $J_{O_{i}}$ is called the object-to-agent Jacobian matrix, with

$$
J_{O_{i}}(s)=\left[\begin{array}{cc}
I_{3} & -S\left(p_{i O}(s)\right) \\
0_{3 \times 3} & I_{3}
\end{array}\right],
$$

$\forall i \in \mathcal{N}$, which is full-rank due to the grasp rigidity. The grasp matrix $G \in \mathbb{R}^{6 \times 6 N}$ is formed by stacking $J_{O_{i}}(s)^{T}, i \in \mathcal{N}$ as

$$
G(s)=\left[J_{O_{1}}(s)^{T}, \ldots, J_{O_{N}}(s)^{T}\right],
$$

and has full row rank. Let $v(s)=\left[v_{1}(s)^{T}, \ldots, v_{N}(s)^{T}\right]^{T}$ and $h=\left[h_{1}^{T}, \ldots, h_{N}^{T}\right]^{T}$. Equation (5.15) can now be written in the following form:

$$
v(s)=G(s)^{T} v_{O}(s) .
$$

The kineto-statics duality (Siciliano et al. [2010]) together with the grasp rigidity suggest that the generalized forces $h_{O}$ and $h_{i}, i \in \mathcal{N}$ are related by

$$
h_{O}=G(s) h \text {. }
$$

For a given $h_{O}$, the solution for (5.19) can be obtained as

$$
h=G^{\dagger} h_{O}+V h_{I} \text {, }
$$

where $G^{\dagger} \in \mathbb{R}^{6 N \times 6}$ is a right inverse of $G$, the matrix $V \in \mathbb{R}^{6 N \times 6}$ is a matrix whose columns span the null space of $G$, and $h_{I} \in \mathbb{R}^{6}$ is an arbitrary vector and generally represents the vector of internal forces, i.e., the forces that do not contribute to the motion of the object. The choice of $h_{I}$ determines which of the possible solutions of (5.19) is chosen. Note that $G^{\dagger}$ is not unique and different choices of $G^{\dagger}$ result in different load distributions. We will refer to $G^{\dagger}$ as a pseudo-inverse of $G$.

The interaction forces $h$ among the manipulators and the object can be decoupled into motion-inducing $h_{\mathrm{m}}$ and internal forces $h_{\text {int }}$ as $h=h_{\mathrm{m}}+h_{\text {int }}$. The 
internal forces are forces that are exerted by the end-effectors on the object and belong to the nullspace of $G$, i.e., $G h_{\text {int }}=0$. Hence, they do not contribute to the motion of the object and result in internal stresses that might damage the object.

It has been recognized in Walker et al. [1991] that the use of a generic pseudoinverse of $G$ in (5.20) may lead to internal forces even if $h_{I}=0$. To avoid this, $G^{\dagger}$ must be chosen properly, which is the topic of the next chapter.

Next, we write equation (5.4) in vector form as

$$
\tilde{\tau}(s)=\tilde{m}(s) \ddot{s}+\tilde{c}(s) \dot{s}^{2}+\tilde{g}(s)+\tilde{J}^{T}(s) h,
$$

where $\tilde{\tau}=\left[\tau_{1}^{T}, \ldots, \tau_{N}^{T}\right]^{T} \in \mathbb{R}^{\tilde{n}}$, with $\tilde{n}=\sum_{i \in \mathcal{N}} n_{i}, \tilde{m}=\left[m_{1}^{T}, \ldots, m_{N}^{T}\right]^{T} \in \mathbb{R}^{\tilde{n}}, \tilde{c}=$ $\left[c_{1}^{T}, \ldots, c_{N}^{T}\right]^{T} \in \mathbb{R}^{\tilde{n}}, \tilde{g}=\left[g_{1}^{T}, \ldots, g_{N}^{T}\right]^{T} \in \mathbb{R}^{\tilde{n}}$, and $\tilde{J}=\operatorname{diag}\left(J_{1}, \ldots, J_{N}\right) \in \mathbb{R}^{6 N \times \tilde{n}}$. Substituting (5.13) for $h_{O}$ in (5.20) and then the resulting $h$ into (5.21) yields the coupled dynamics

$$
\begin{aligned}
\tilde{\tau}(s)= & \left(\tilde{m}(s)+\tilde{J}^{T}(s) G^{\dagger}(s) m_{O}(s)\right) \ddot{s} \\
& +\left(\tilde{c}(s)+\tilde{J}^{T}(s) G^{\dagger}(s) c_{O}(s)\right) \dot{s}^{2} \\
& +\tilde{g}(s)+\tilde{J}^{T}(s) G^{\dagger}(s) g_{O}+\tilde{J}^{T}(s) V(s) h_{I} .
\end{aligned}
$$

The above equation describes the coupled dynamics of $N$ robotic manipulators rigidly grasping an object, and is expressed in terms of $\ddot{s}, \dot{s}^{2}$ and $s$.

Finally, using an argument and a procedure similar to those employed in Chapter 4, the problem of interest in this chapter can be cast as a convex optimization problem. This involves using $a(s)=\ddot{s}$ and $b(s)=\dot{s}^{2}$ as optimization variables. As a result of this change of variables, the coupled dynamics (5.22) which enters as constraints in the optimization problem, becomes linear in $a$ and $b$.

The problem of interest can be formulated as the following convex optimization problem:

$$
\begin{aligned}
& \underset{a(\cdot), b(\cdot), \tilde{\tau}(\cdot)}{\operatorname{minimize}} \int_{0}^{1} \frac{1}{\sqrt{b(s)}} d s \\
& \text { subject to } \quad \tilde{\tau}(s)=\left(\tilde{m}(s)+\tilde{J}^{T}(s) G^{\dagger}(s) m_{O}(s)\right) a(s) \\
& +\left(\tilde{c}(s)+\tilde{J}^{T}(s) G^{\dagger}(s) c_{O}(s)\right) b(s) \\
& +\tilde{g}(s)+\tilde{J}^{T}(s) G^{\dagger}(s) g_{O}+\tilde{J}^{T}(s) V(s) h_{I}, \\
& b(0)=\dot{s}_{0}^{2} \text {, } \\
& b(1)=\dot{s}_{T}^{2} \text {, } \\
& b(s) \geq 0 \text {, } \\
& b(s) \leq \bar{b}(s) \text {, } \\
& b^{\prime}(s)=2 a(s) \text {, } \\
& \underline{\tau}_{i}(s) \leq \tau_{i}(s) \leq \bar{\tau}_{i}(s), \quad i \in \mathcal{N},
\end{aligned}
$$




$$
\forall s \in[0,1]
$$

where $\underline{\tau}_{i}$ and $\bar{\tau}_{i}$ are the lower and upper bounds on the $i$ th manipulator's joint torques, respectively. This problem can finally be reformulated as an SOCP using a procedure similar to the one used in Chapter 3.

\subsection{Numerical simulation}

In this section, we provide some numerical simulations to illustrate the results. Consider the problem setup described in Section 5.1 with two three-link planar manipulators. Suppose the manipulators are rigidly grasping a cuboid object of dimensions $0.07 \times 0.07 \times 0.4 \mathrm{~m}^{3}$ and mass $12 \mathrm{~kg}$, and suppose the centre of mass of the object is located at its centroid. The dynamic parameters used for the simulation are given in Table 5.1, where $m_{i j}$ and $l_{i j}$ denote the mass and the length of the $j$ th link of the $i$ th manipulator, respectively. We assume that each link is a cylinder of radius $0.025 \mathrm{~m}$ and compute the inertia tensors accordingly.

Table 5.1: Dynamic parameters

\begin{tabular}{|l|ccc|ccc|}
\hline & $m_{i 1}(\mathrm{~kg})$ & $m_{i 2}(\mathrm{~kg})$ & $m_{i 3}(\mathrm{~kg})$ & $l_{i 1}(\mathrm{~m})$ & $l_{i 2}(\mathrm{~m})$ & $l_{i 3}(\mathrm{~m})$ \\
\hline First manipulator $(i=1)$ & 12 & 10 & 1.5 & 0.8 & 0.7 & 0.1 \\
\hline Second manipulator $(i=2)$ & 9 & 9 & 1.5 & 0.6 & 0.6 & 0.1 \\
\hline
\end{tabular}

The desired path for the centre of mass of the object is given by

$$
p_{O}(s)=\left[\begin{array}{c}
0.23-0.12 \sin (\pi s) \\
0.35+0.65 s \\
0
\end{array}\right]
$$

For the orientation of the object, we have used (5.1) with the roll-pitch-yaw angles $\phi_{O_{i}}=\left[\begin{array}{lll}\pi / 6 & 0 & 0\end{array}\right]^{T}$ and $\phi_{O_{f}}=\left[\begin{array}{lll}0 & 0 & 0\end{array}\right]^{T}$ for the initial and the final orientations, respectively. Figure 5.2 shows the path for the object's centre of mass as well as the postures of the manipulators in steps of 0.037 .

The pseudo-inverse $G^{\dagger}$ and the matrix $V$ used in this simulation are as follows (see Siciliano and Khatib [2016]):

$$
G^{+}=\frac{1}{2}\left[\begin{array}{cc}
I_{3} & 0_{3 \times 3} \\
-S\left(p_{10}\right) & I_{3} \\
I_{3} & 0_{3 \times 3} \\
-S\left(p_{20}\right) & I_{3}
\end{array}\right], \quad V=\left[\begin{array}{cc}
-I_{3} & 0_{3 \times 3} \\
S\left(p_{10}\right) & -I_{3} \\
I_{3} & 0_{3 \times 3} \\
-S\left(p_{20}\right) & I_{3}
\end{array}\right] \text {, }
$$

and $h_{I}$ is set to zero.

For the simulation, the path coordinate $s$ is discretized on 100 grid points $(K=99)$. The optimization problem is implemented and solved in SOCP form in MATLAB using the optimization modeling toolbox YALMIP (Lofberg [2004]) and 


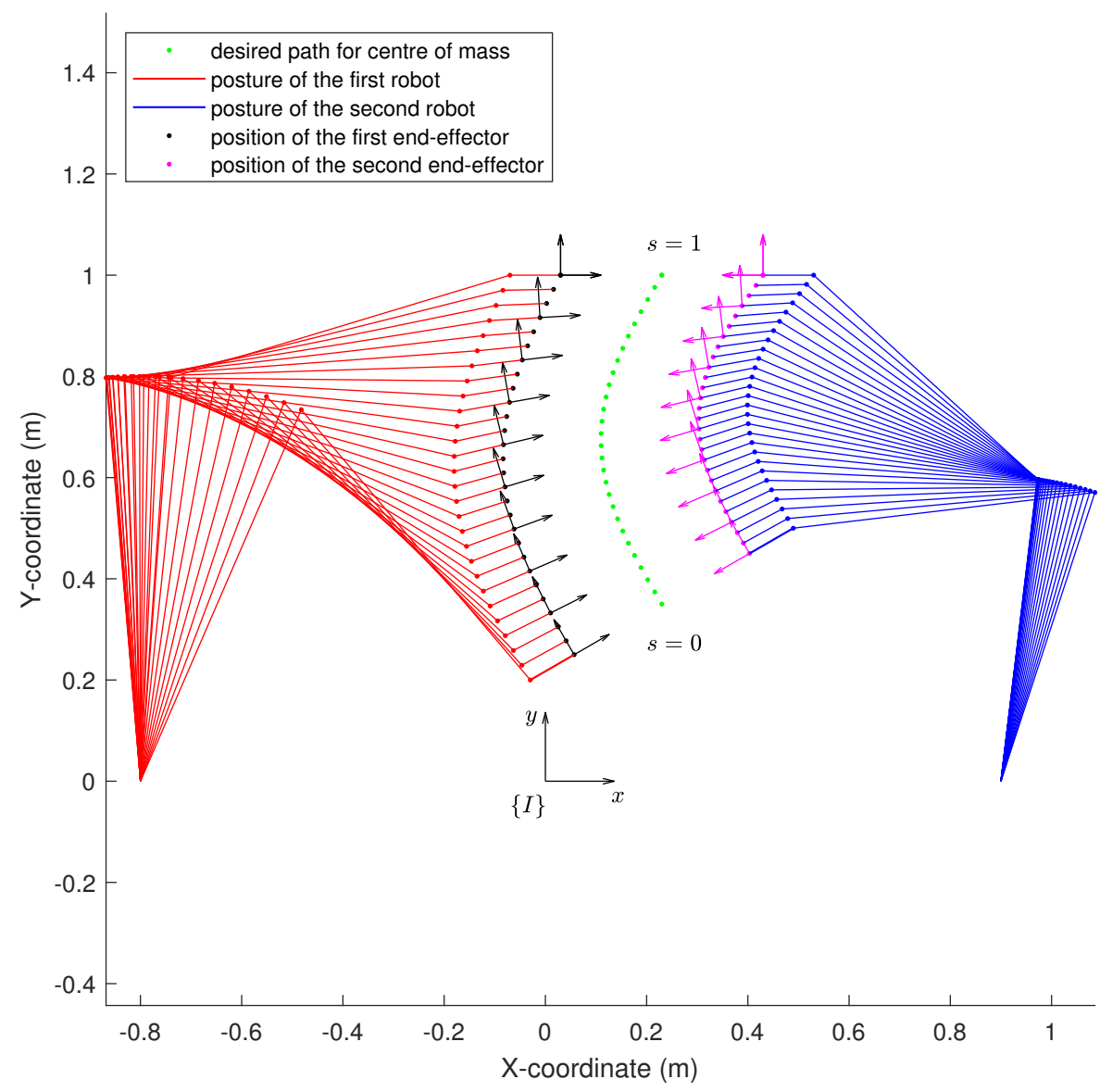

Figure 5.2: Desired path for the centre of mass of the object, together with the postures of the manipulators during the motion. Links of the first and second manipulators are shown by red and blue lines, respectively. The frames colored in black and magenta show the orientations of the first and second end-effectors, respectively. 
the solver MOSEK (ApS [2021]). The simulation is performed on a laptop with a 2.6 GHz Intel Core i7-5600U processor.

The approach presented in this chapter results in a minimal traversal time of $0.660 \mathrm{sec}$ for this simulation. Figure 5.3 shows the resulting joint torques and the corresponding bounds for both manipulators as functions of the path coordinate $s$. Once the optimization variables $a$ and $b$ are obtained, equations (5.3a) and (5.3b) can be used to compute the joint velocities and accelerations, respectively. Figure 5.4 shows the evolution of the joint velocities with respect to the path coordinate $s$ for both manipulators, where the initial and the final velocities have been set to zero. The considered bounds on the joint velocities can be seen in the same figure.

From the plots it can be seen that for every point along the path, either one of the joint torques of the first or second manipulator is in saturation, or one of the joint velocities has reached its bound. This is in line with the findings in the work by Chen [1990], where it is shown that the minimum-time control for multiple manipulators requires that at least one of the actuators is always saturated on any finite time subinterval, while the rest of them adjust their torques so that other constraints on the motion are not violated. 

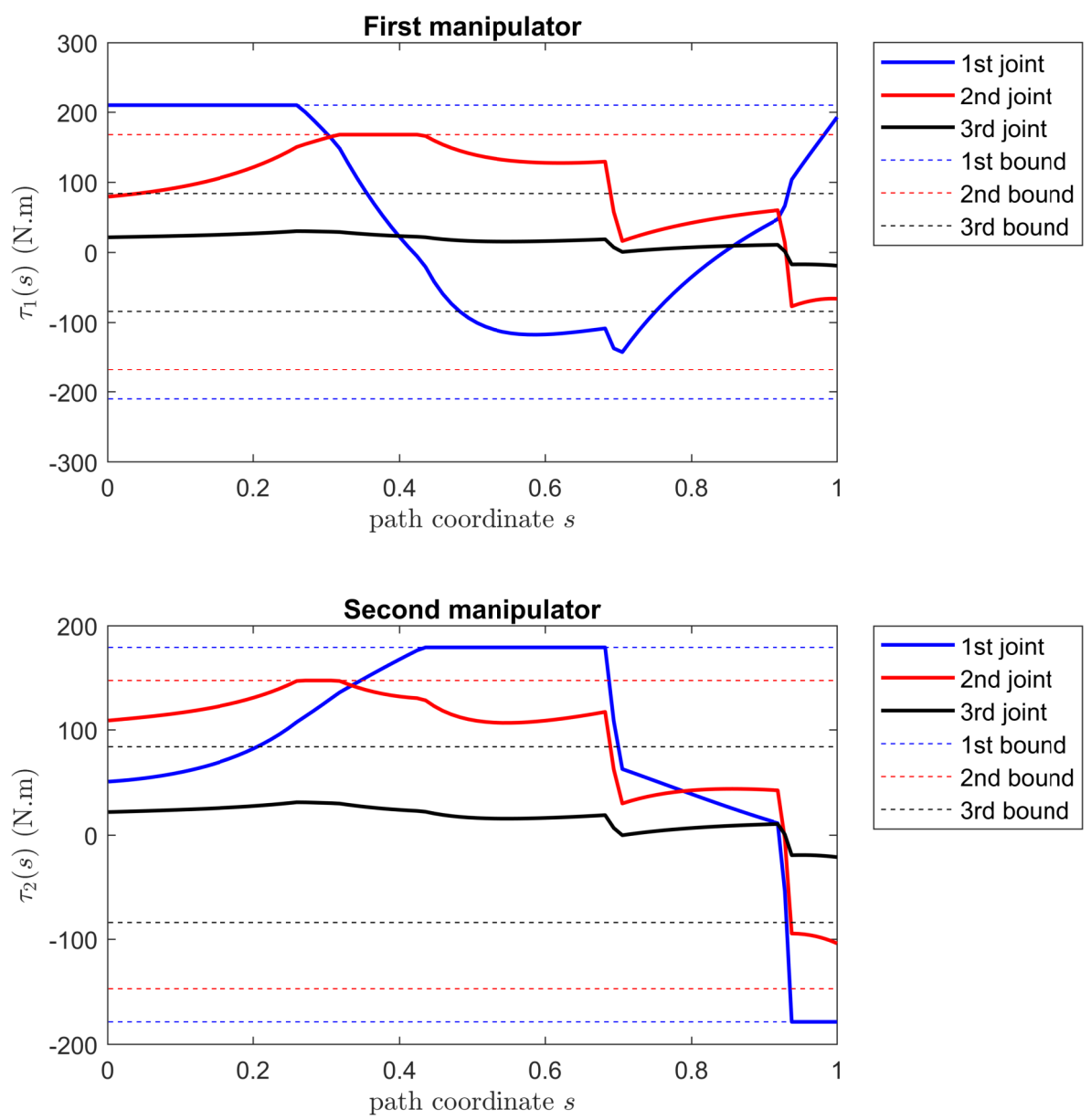

Figure 5.3: Joint torques $\tau_{1}(s)$ and $\tau_{2}(s)$ 

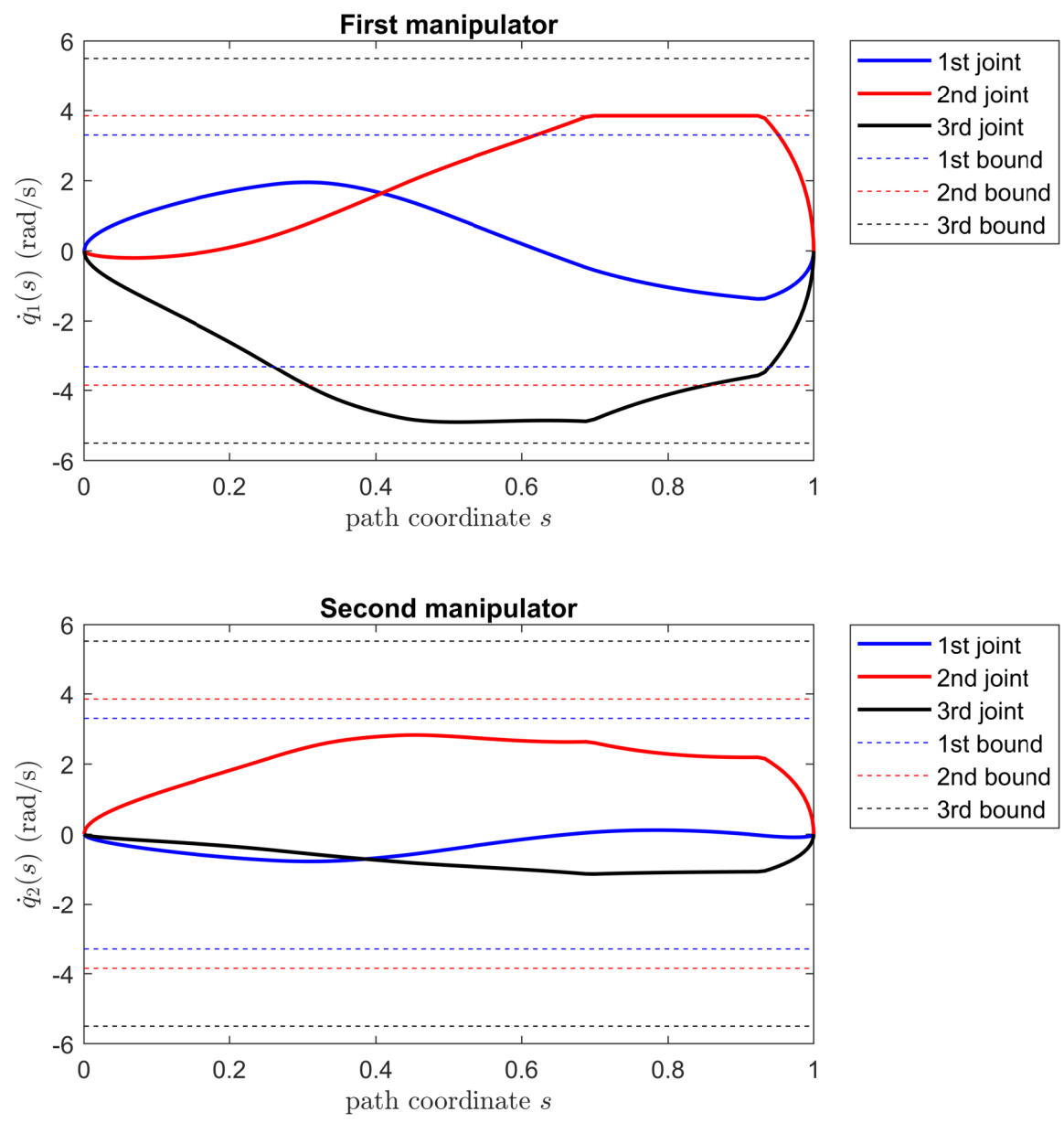

Figure 5.4: Joint velocities $\dot{q}_{1}(s)$ and $\dot{q}_{2}(s)$ 



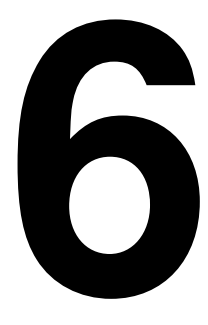

\section{Internal force-free cooperative path tracking}

In this chapter, a new approach to obtain load distributions free of internal forces is proposed. In this approach, the object is divided into several parts and the forces required to move the parts according to the desired motion of the object are compared with the forces resulting from a load distribution strategy. The result is a new pseudo-inverse of the grasp matrix that leads to internal force-free distributions. This pseudo-inverse is parameterized by coefficients that have the meaning of the inertial parameters of the considered parts of the object. The results are further investigated for scenarios with three-dimensional convex object with uniform mass density. The proposed pseudo-inverse can be combined with the results in Chapter 5 to solve the problem of time-optimal cooperative path tracking subject to zero internal forces during the motion.

The next section gives an introduction to the chapter and reviews the related work. The problem of interest is stated in Section 6.2. The main results of the chapter are presented in Section 6.3. Section 6.4 contains some simulations to illustrate the use of the obtained pseudo-inverse in the time-optimal cooperative path tracking problem.

\subsection{Introduction}

In a cooperative manipulation task, in general there exists an infinite number of generalized forces exerted by the end-effectors that result in the same desired generalized forces on the centre of mass of the object. This is due to multiple manipulators being involved in the manipulation task. This redundancy needs to be resolved using a load distribution strategy. The resulting interaction forces among the manipulators and the object from a load distribution strategy, can be decomposed into motion-inducing and internal forces. The internal forces belong to the nullspace of the grasp matrix (Walker et al. [1991]; Erhart and 
Hirche [2015]) and do not contribute to the motion of the manipulated object. While a certain amount of such forces can be useful in some cases (e.g., in order to ensure a firm grasp), in general they must be avoided to prevent object damage and unnecessary effort of the manipulators.

In continuum mechanics, internal stress is defined as the contact forces between neighbouring particles inside an object. Within the scope of this thesis, we are not interested in finding the actual internal stress inside a manipulated object. Internal stress occurs even when an object is grasped by only one manipulator and cannot be avoided. In the scope of cooperative multi-robot manipulation, internal forces are considered to be squeezing (or pulling) forces that manipulators exert to the object and do not contribute to the motion of the object.

As seen in Chapter 5, the load distribution in a cooperative manipulation task is tightly connected to the choice of the pseudo-inverse of the grasp matrix. It has been recognized in Walker et al. [1991] that the use of a generic pseudo-inverse of the grasp matrix may lead to internal forces when $h_{I}=0$ in (5.20). The same work proposes a specific pseudo-inverse in order to avoid the internal forces. The work by Chung et al. [2005] challenged the results for the pseudo-inverse proposed by Walker et al. [1991] and suggested to use the Moore-Penrose pseudo-inverse instead. In the works by Kumar and Waldron [1988] and Bonitz and Hsia [1994], the absence of internal forces is considered to be equivalent to the difference between any two end-effector forces projected along the line joining the two contact points being zero. It is, however, not clear how this concept accounts for internal torques. The work by Erhart and Hirche [2015] characterizes the internal forces as the violation of the kinematic constraints imposed on the ensemble of the manipulators, and provides a parameterized pseudo-inverse of the grasp matrix for obtaining load distributions free of internal forces. This suggests that the load distributions free of internal forces are not unique and there can be infinitely many load distributions of this type. The work by Erhart and Hirche [2015] finds some of these load distributions. The work by Verginis et al. [2019] studies the rigid cooperative manipulation using rigidity theory and proposes a new pseudo-inverse in order to have a internal force-free load distribution. In summary, finding all load distributions that are free of internal forces is still an open issue.

In this chapter, we propose a novel approach to obtain load distributions free of internal forces, which results in a parameterized pseudo-inverse different from the one proposed by Erhart and Hirche [2015]. Compared to the work in Erhart and Hirche [2015], our approach is more intuitive, since it is based on dividing the object into several segments and the parameter used for the load distributions have the meaning of the inertial parameters of these segments. Furthermore, in our approach it is clear how to assign more load to a particular manipulator, whereas in Erhart and Hirche [2015] it is not straightforward how to do this. In most of the aforementioned works, for example the recent work by Verginis et al. [2019], in order to have a internal force-free distribution a particular pseudoinverse is proposed which leads to a particular load distribution, whereas the derived pseudo-inverse from our approach offers different load distributions, all free of internal forces. This is achieved by changing the parameters of the proposed pseudo-inverse. The possibility of having different load distributions free 


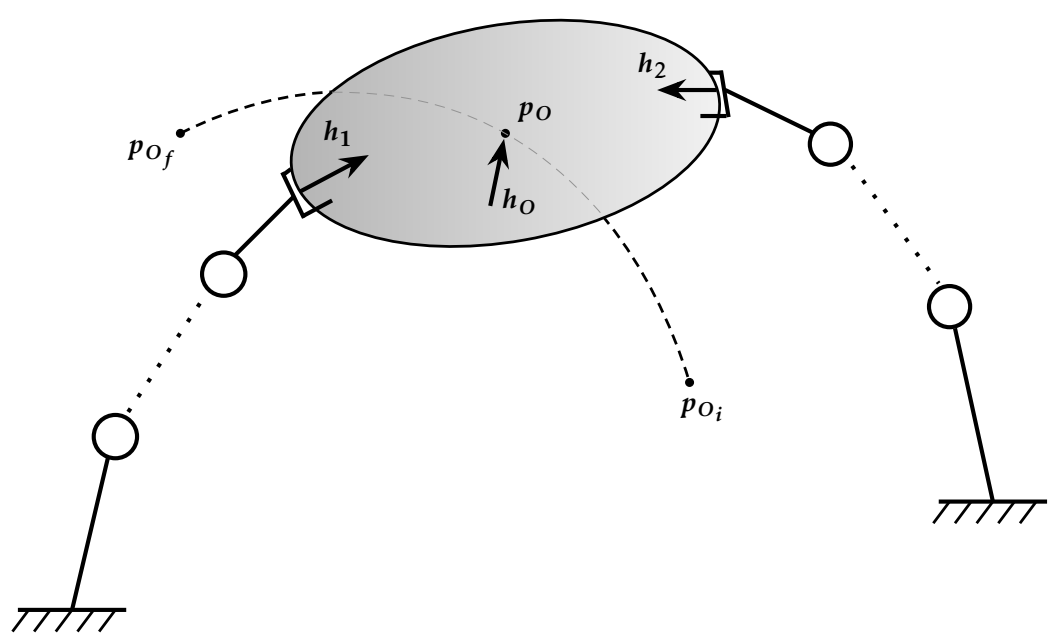

Figure 6.1: Two robotic manipulators rigidly grasping an object. The points $p_{O_{i}}$ and $p_{O_{f}}$ are the initial and the final positions of the given path, respectively.

of internal forces can be exploited to account for the differences in the capabilities of manipulators in a cooperative manipulation task.

\subsection{Problem statement}

This section states the problem of interest for completeness. Let us consider the problem setup described in Section 5.1. As mentioned in Chapter 5, the use of a generic pseudo-inverse of $G$ in (5.20) might still lead to internal forces when $h_{I}=0$. To avoid this, $G^{\dagger}$ must be chosen properly. This chapter concerns finding a suitable pseudo-inverse of $G$ so that resulting force distribution from the first term in (5.20), i.e., $G^{\dagger} h_{O}$, becomes free of internal forces.

\subsection{Main results}

In this section, we provide the main result of the chapter. First, the idea behind our approach is presented. For simplicity, we consider two robotic manipulators rigidly grasping an object as in Figure 6.1. We call this case the cooperative case. The extension of the results that follow to a scenario with $N$ manipulators is straightforward.

Suppose the object is divided into two separate parts, indexed by $i \in\{1,2\}$, and the $i$ th part is rigidly grasped by the $i$ th manipulators, see Figure 6.2. We assume that there is an infinitesimal distance between the pieces and, in turn, we assume that there is no interaction and exchange of forces between them. We 


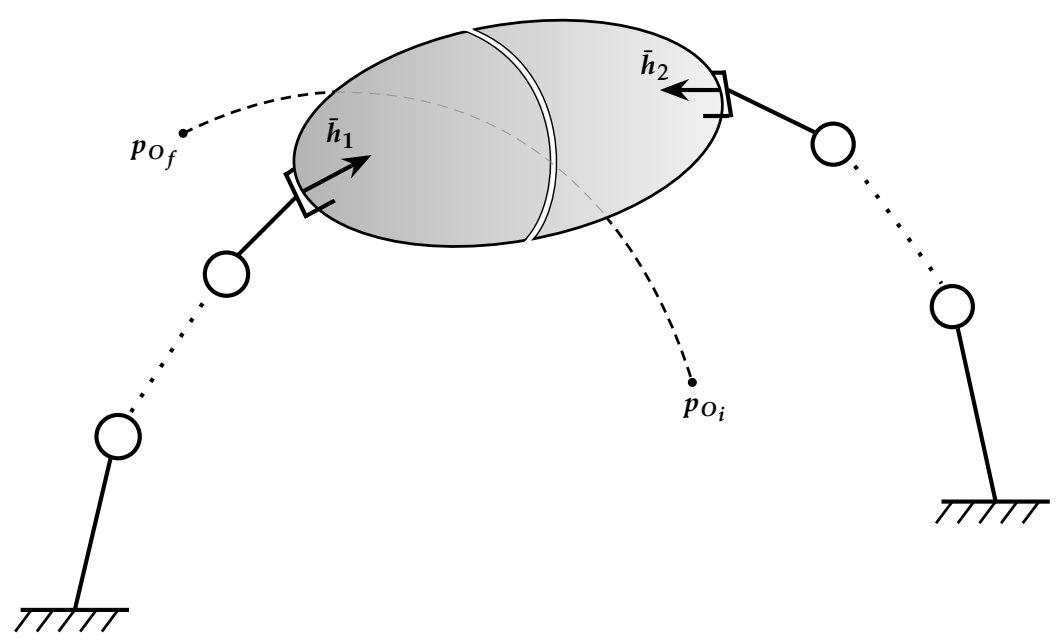

Figure 6.2: Object divided into two parts. Each part is rigidly grasped by one manipulator.

want each segment to be moved in precisely the same way that the corresponding part of the object would move in the cooperative case. That is, the position and orientation displacement of a frame attached to each segment is zero with respect to a frame attached to the other segment; each segment moves along the path corresponding to that segment from the cooperative case; the velocities and accelerations of each segment during the motion are the same as those that the corresponding part of the object would experience in the cooperative case; and $\omega_{O_{i}}=\omega_{O}, i \in\{1,2\}$, where $\omega_{O_{i}} \in \mathbb{R}^{3}$ and $\omega_{O} \in \mathbb{R}^{3}$ are the angular velocities of the $i$ th segment and the object in cooperative case, respectively.

Let $\bar{h}_{i} \in \mathbb{R}^{6}, i \in\{1,2\}$ denote the generalized forces exerted by the $i$ th endeffector to the $i$ th segment at the grasping point. Once the desired motion of each segment is specified, the generalized forces $\bar{h}_{i}$ required to fulfill the motion can be obtained. In this setup, since each segment of the object is grasped by only one manipulator and no forces are exchanged between the segments, there are no internal forces by design. Employing the generalized forces $\bar{h}_{i}$ in the cooperative case will not result in internal forces, since the characteristics of the segments together are the same as the characteristics of the whole object, and also the imposed kinematic constraints in both cases are the same. Hence, the obtained generalized forces $\bar{h}_{i}, i \in\{1,2\}$, are a distribution of forces that employing them in the cooperative case will move the object along the given path with specified orientation, velocities and accelerations, and without resulting in internal forces.

The next step would be to find a pseudo-inverse of $G$ that results in the same distribution of forces mentioned above, i.e., finding the pseudo-inverse 


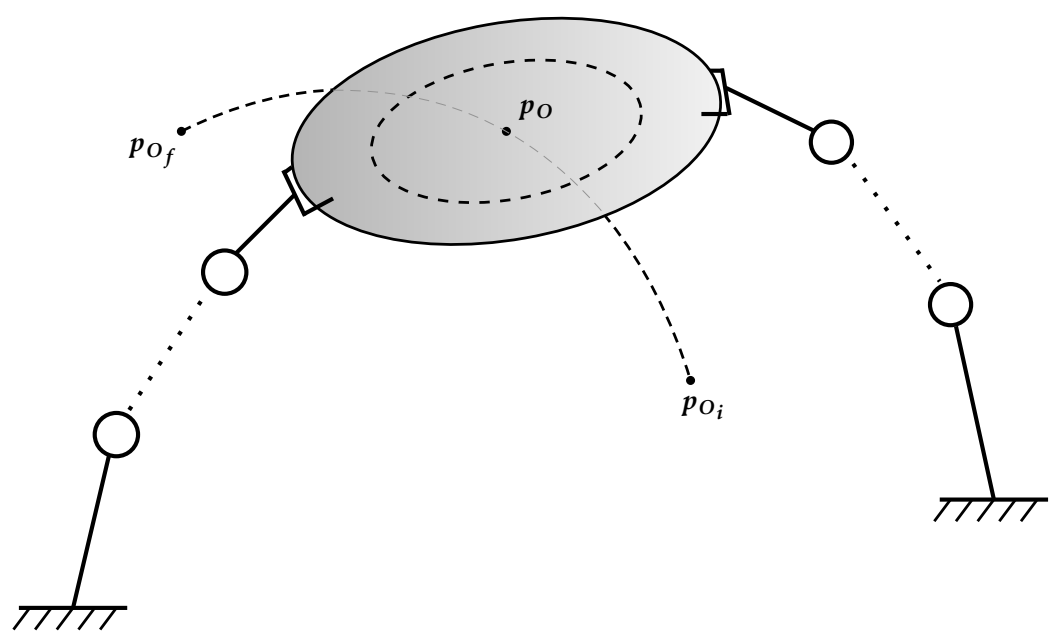

Figure 6.3: Segments are chosen in such a way that their centres of mass become the same as the object's centre of mass. The boundary of the first segment is shown by the dashed line. The second segment is obtained by excluding the first one.

$G^{\star} \in \mathbb{R}^{12 \times 6}$ such that the resulting generalized forces $h_{i}, i \in\{1,2\}$ from

$$
h=G^{\star} h_{\mathrm{O}},
$$

become the same as $\bar{h}_{i}, i \in\{1,2\}$, respectively.

Choosing the segments as in Figure 6.2, where their centres of mass are different from the centre of mass of the whole object, makes the problem of finding the pseudo-inverse $G^{\star}$ intractable. The reason for this is explained later in this section. To resolve this problem, we choose the segments in such a way that their centres of mass become the same as the object's centre of mass, see Figure 6.3 for an illustration. Note that this segmentation is imaginary. In Figure 6.3, one can see that the inner segment is not directly grasped by the manipulators. Although this is different from the setup described above, the above arguments still hold. This is because the segments serve to obtain the generalized forces $\bar{h}_{i}$ required to move the segments in a desired way, assuming that they behave as if they are rigidly grasped. This is the case for any imaginary shape on the object, including the considered segments in Figure 6.3, since the whole object is rigidly grasped and the object itself is rigid.

Having discussed the idea behind our approach, we next find the pseudoinverse $G^{\star}$.

Let us consider the $i$ th segment and the $i$ th manipulator in Figure 6.3. Let $x_{O_{i}} \in \mathbb{R}^{6}$ and $v_{O_{i}} \in \mathbb{R}^{6}$ denote the pose and the velocity of the segment's centre of mass, respectively. Since the segments are chosen in such a way that their centres of mass are the same as the object's centre of mass, and since $\omega_{O_{i}}=\omega_{0}$, we get 
$v_{O_{i}}=v_{O}$ where $v_{O}$ is the velocity of the object's centre of mass. Replacing $v_{O_{i}}$ with $v_{O}$, the second-order dynamics of the $i$ th segment is given by

$$
M_{O_{i}}\left(x_{O_{i}}\right) \dot{v}_{O}+C_{O_{i}}\left(x_{O_{i}}, \dot{x}_{O_{i}}\right) v_{O}+g_{O_{i}}=h_{O_{i}},
$$

where

$$
\begin{aligned}
M_{O_{i}} & =\left[\begin{array}{cc}
m_{O_{i}} I_{3} & 0_{3 \times 3} \\
0_{3 \times 3} & I_{O_{i}}
\end{array}\right], \\
C_{O_{i}} & =\left[\begin{array}{cc}
0_{3 \times 3} & 0_{3 \times 3} \\
0_{3 \times 3} & S\left(\omega_{O_{i}}\right) I_{O_{i}}
\end{array}\right], \\
g_{O_{i}} & =\left[\begin{array}{c}
-m_{O_{i}} g \\
0_{3}
\end{array}\right],
\end{aligned}
$$

and where $m_{O_{i}}$ is the mass of the $i$ th segment, $I_{O_{i}} \in \mathbb{R}^{3 \times 3}$ is the $i$ th segment's inertia tensor relative to the centre of mass when expressed in a frame parallel to $\{I\}$ with origin at the centre of mass, and $h_{O_{i}} \in \mathbb{R}^{6}$ is the vector of generalized forces acting on the centre of mass of the $i$ th segment. Note that in our setup, $h_{O_{i}}$ is contributed by the $i$ th manipulator.

Next, we obtain the generalized forces $\bar{h}_{i}, i \in\{1,2\}$. From (5.19), the relationship between the generalized forces $h_{O_{i}}$ and $\bar{h}_{i}$ can be written as

$$
h_{O_{i}}=G_{i} \bar{h}_{i} \text {, }
$$

where $G_{i} \in \mathbb{R}^{6 \times 6}$ is given by

$$
G_{i}=J_{O_{i}}^{T}=\left[\begin{array}{cc}
I_{3} & 0_{3 \times 3} \\
S\left(p_{i O}\right) & I_{3}
\end{array}\right] .
$$

From (6.4), we have $\bar{h}_{i}=G_{i}^{-1} h_{O_{i}}$. Substituting (6.2) for $h_{O_{i}}$ in this equation yields

$$
\bar{h}_{i}=\left(G_{i}^{-1} M_{O_{i}}\right) \dot{v}_{O}+\left(G_{i}^{-1} C_{O_{i}}\right) v_{O}+G_{i}^{-1} g_{O_{i}}, \quad i \in\{1,2\},
$$

where the inverse matrix of $G_{i}$ can be computed as

$$
G_{i}^{-1}=\left[\begin{array}{cc}
I_{3} & 0_{3 \times 3} \\
-S\left(p_{i O}\right) & I_{3}
\end{array}\right] .
$$

In addition, substituting (5.6) in (6.1) yields

$$
h_{i}=\left(G_{i}^{\star} M_{O}\right) \dot{v}_{O}+\left(G_{i}^{\star} C_{O}\right) v_{O}+G_{i}^{\star} g_{O}, \quad i \in\{1,2\},
$$

where we have denoted and used $G^{\star}=\left[G_{1}^{\star T}, G_{2}^{\star^{T}}\right]^{T}$. The goal is then to choose $G_{i}^{\star} \in \mathbb{R}^{6 \times 6}, i \in\{1,2\}$ so that $(6.8)$ becomes equal to $(6.6)$ for every $v_{O}$ and $\dot{v}_{O}$ that the object might experience. This means that $G_{i}^{\star}, i \in\{1,2\}$ must satisfy the following equations:

$$
\begin{aligned}
G_{i}^{\star} M_{O} & =G_{i}^{-1} M_{O_{i}}, \\
G_{i}^{\star} C_{O} & =G_{i}^{-1} C_{O_{i}}, \\
G_{i}^{\star} g_{O} & =G_{i}^{-1} g_{O_{i}} .
\end{aligned}
$$


From $(6.9 \mathrm{c})$ and $(6.9 \mathrm{a}), G_{i}^{\star}$ can be obtained as

$$
G_{i}^{\star}=\left[\begin{array}{cc}
\frac{m_{O_{i}}}{m} I_{3} & 0_{3 \times 3} \\
-\frac{m_{O_{i}}}{m} S\left(p_{i O}\right) & I_{O_{i}} I_{O}^{-1}
\end{array}\right] .
$$

Now, it remains to be checked whether this matrix satisfies $(6.9 \mathrm{~b})$. Since $\omega_{O_{i}}=$ $\omega_{O}, i \in\{1,2\}$, the right-hand side of $(6.9 \mathrm{~b})$ is equal to

$$
G_{i}^{-1} C_{O_{i}}=\left[\begin{array}{cc}
0_{3 \times 3} & 0_{3 \times 3} \\
0_{3 \times 3} & S\left(\omega_{O}\right) I_{O_{i}}
\end{array}\right] .
$$

Using (6.10), the left-hand side of $(6.9 \mathrm{~b})$ becomes

$$
G_{i}^{\star} C_{O}=\left[\begin{array}{cc}
0_{3 \times 3} & 0_{3 \times 3} \\
0_{3 \times 3} & I_{O_{i}} I_{O}^{-1} S\left(\omega_{O}\right) I_{O}
\end{array}\right] .
$$

For (6.11) and (6.12) to be equal, the following equation must hold:

$$
I_{O_{i}} I_{O}^{-1} S\left(\omega_{O}\right) I_{O}=S\left(\omega_{O}\right) I_{O_{i}}
$$

It can be easily seen that this equation holds if the segments can be chosen in such a way that their inertia tensors are fractions of the object's inertia tensor. In other words

$$
I_{O_{i}}=\lambda_{i} I_{O}, \quad i \in\{1,2\},
$$

where $\lambda_{i}, i \in\{1,2\}$ are some positive coefficients with $\lambda_{1}+\lambda_{2}=1$. Note that $\lambda_{1}+\lambda_{2}=1$ follows from the fact that the sum of the inertia tensors of all the segments is equal to the object's inertia tensor, i.e., $I_{\mathrm{O}_{1}}+I_{\mathrm{O}_{2}}=I_{O}$. The coefficients $\lambda_{i}, i \in\{1,2\}$, are generally functions of the mass ratios between the segments and the whole object.

As mentioned earlier, if the segments are chosen in such a way that their centres of mass are different from the centre of mass of the object, the problem of finding the pseudo-inverse $G^{\star}$ becomes intractable. This can be seen by following the procedure described above to obtain the pseudo-inverse for such a scenario. If the segments are chosen as in Figure 6.2 and the above-mentioned steps are performed, instead of equation (6.13) we end up with a complicated equation for which it is difficult to find a solution similar to (6.14).

We proceed by investigating the obtained results for convex objects. For a convex object with uniform mass density, the boundaries of the segments satisfying (6.14) can be obtained by shrinking the object with the same ratio in all directions, see Figure 6.4 for an illustration. Let $\bar{\lambda}_{i}$ be such a ratio corresponding to the $i$ th segment. In other words, for obtaining the boundary of the $i$ th segment, a point on the boundary of the object with the spherical coordinates $(r, \theta, \varphi)$ is mapped to a point with the spherical coordinates $\left(\bar{\lambda}_{i} r, \theta, \varphi\right)$. Here, $r$ is the distance to the origin which is the same as the centre of mass, and $\theta$ and $\varphi$ are azimuthal and polar angles, respectively.

Next, we compute the coefficients $\lambda_{i}, i \in\{1,2\}$ for three-dimensional convex objects with uniform mass density. Consider a three-dimensional convex object 


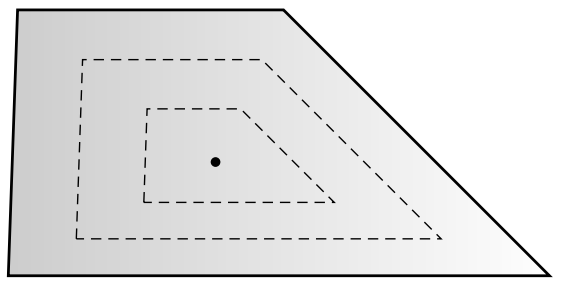

Figure 6.4: An example of segments satisfying (6.14) for a two-dimensional convex object grasped by three manipulators. The dashed lines are the boundaries of the segments and the point is the object's centre of mass.

with the constant mass density $\rho$. The inertia tensor in the object reference frame $\{O\}$ with origin at the centre of mass is computed as (Spong et al. [2006])

$$
I_{O}^{O}=\left[\begin{array}{ccc}
I_{O_{x x}}^{O} & I_{O_{x y}}^{O} & I_{O_{x z}}^{O} \\
I_{O_{y x}}^{O} & I_{O_{y y}}^{O} & I_{O_{y z}}^{O} \\
I_{O_{z x}}^{O} & I_{O_{z y}}^{O} & I_{O_{z z}}^{O}
\end{array}\right],
$$

where

$$
\begin{aligned}
& I_{O_{x x}}^{O}=\iiint_{D_{O}}\left(y^{2}+z^{2}\right) \rho d x d y d z, \\
& I_{O_{y y}}^{O}=\iiint_{D_{O}}\left(x^{2}+z^{2}\right) \rho d x d y d z, \\
& I_{O_{z z}}^{O}=\iiint_{D_{O}}\left(x^{2}+y^{2}\right) \rho d x d y d z, \\
& I_{O_{x y}}^{O}=I_{O_{y x}}^{O}=-\iiint_{D_{O}} x y \rho d x d y d z, \\
& I_{O_{x z}}^{O}=I_{O_{z x}}^{O}=-\iiint_{D_{O}} x z \rho d x d y d z, \\
& I_{O_{y z}}^{O}=I_{O_{z y}}^{O}=-\iiint_{D_{O}} y z \rho d x d y d z .
\end{aligned}
$$

The integrals in the above expressions are computed over the region of space occupied by the object, denote by $D_{O}$. Note that the inertia tensor (6.15) is a symmetric, positive definite matrix. Similarly, let $I_{O_{i}}^{O}$ be the $i$ th segment's inertia 
tensor in the object reference frame $\{O\}$, with

$$
I_{O_{i}}^{O}=\left[\begin{array}{lll}
I_{O_{i_{x x}}}^{O} & I_{O_{i_{x y}}}^{O} & I_{O_{i_{x z}}}^{O} \\
I_{O_{i_{y x}}}^{O} & I_{O_{i_{y y}}}^{O} & I_{O_{i_{y z}}}^{O} \\
I_{O_{i_{z x}}}^{O} & I_{O_{i_{z y}}}^{O} & I_{O_{i_{z z}}}^{O}
\end{array}\right],
$$

where the elements are obtained similar to (6.16) with the integration over the region of space occupied by the $i$ th segment. Let us denote this region of space by $D_{O_{i}}$.

By changing the integration variables from $(x, y, z)$ to $\left(x_{1}, y_{1}, z_{1}\right)=\left(\bar{\lambda}_{1} x, \bar{\lambda}_{1} y, \bar{\lambda}_{1} z\right)$, the first diagonal element of $I_{O}^{O}$ can be written as

$$
\begin{aligned}
I_{O_{x x}}^{O}=\iiint_{D_{O}}\left(y^{2}+z^{2}\right) \rho d x d y d z & =\iiint_{D_{O_{1}}}\left(\frac{1}{\bar{\lambda}_{1}^{2}} y_{1}^{2}+\frac{1}{\bar{\lambda}_{1}^{2}} z_{1}^{2}\right) \rho \frac{1}{\bar{\lambda}_{1}^{3}} d x_{1} d y_{1} d z_{1} \\
& =\frac{1}{\bar{\lambda}_{1}^{5}} \iiint_{D_{O_{1}}}\left(y_{1}^{2}+z_{1}^{2}\right) \rho d x_{1} d y_{1} d z_{1}, \\
& =\frac{1}{\bar{\lambda}_{1}^{5}} I_{O_{1_{x x}}}^{O} .
\end{aligned}
$$

Note that by the above-mentioned change of variables, the integration region changes from $D_{O}$ to $D_{O_{1}}$. This can easily be seen in spherical coordinates. Similar relationships can be obtained between the other elements of $I_{O}^{O}$ and $I_{O_{1}}^{O}$. As a result, we get $I_{O_{1}}^{O}=\bar{\lambda}_{1}^{5} I_{O}^{O}$, from which it follows that

$$
I_{O_{1}}=\bar{\lambda}_{1}^{5} I_{O}
$$

Next, we find the relationship between the masses of the first segment and the whole object. The mass of the object is given by

$$
m=\iiint_{D_{O}} \rho d x d y d z
$$

which, by changing the integration variables from $(x, y, z)$ to $\left(x_{1}, y_{1}, z_{1}\right)$ can be written as

$$
m=\iiint_{D_{O}} \rho d x d y d z=\iiint_{D_{O_{1}}} \rho \frac{1}{\bar{\lambda}_{1}^{3}} d x_{1} d y_{1} d z_{1}=\frac{1}{\bar{\lambda}_{1}^{3}} m_{O_{1}} .
$$

From (6.19) and (6.21), and noting that $I_{O_{1}}+I_{O_{2}}=I_{O}$, the coefficients $\lambda_{i}, i \in\{1,2\}$ can be obtained as

$$
\begin{aligned}
& \lambda_{1}=\left(\frac{m_{O_{1}}}{m}\right)^{\frac{5}{3}}, \\
& \lambda_{2}=1-\left(\frac{m_{O_{1}}}{m}\right)^{\frac{5}{3}} .
\end{aligned}
$$


In conclusion, a pseudo-inverse of the form

$$
G^{\star}=\left[\begin{array}{cc}
\frac{m_{O_{1}}}{m} I_{3} & 0_{3 \times 3} \\
-\frac{m_{O_{1}}}{m} S\left(p_{1 O}\right) & \lambda_{1} I_{3} \\
\frac{m_{O_{2}}}{m} I_{3} & 0_{3 \times 3} \\
-\frac{m_{O_{2}}}{m} S\left(p_{2 O}\right) & \lambda_{2} I_{3}
\end{array}\right],
$$

with $\lambda_{i}, i \in\{1,2\}$ being given by (6.22) for three-dimensional convex objects with uniform mass density, results in force distributions free of internal forces. It can be readily checked that $G G^{\star}=I_{6}$ holds. Note that the mass ratios $m_{O_{1}} / m$ and $m_{\mathrm{O}_{2}} / m=1-m_{\mathrm{O}_{1}} / m$ are chosen by the user. This freedom allows to account for the potential differences in the power capabilities of the manipulators.

The results presented here straightforwardly extend to a scenario with $N$ manipulators.

Now, let us consider the problem of interest in Chapter 5 with an additional requirement that there must be no internal forces acting on the object. The corresponding optimization problem can be formulated as the convex optimization problem (5.23), with the difference that $h_{I}$ should be set to zero and the pseudoinverse $G^{\dagger}$ must be chosen to be $G^{\star}$. The freedom in the choice of the mass ratios in $(6.23)$ can be exploited to further optimize the problem.

\subsection{Numerical simulation}

In this section, we illustrate the use of the proposed pseudo-inverse in the timeoptimal cooperative path tracking problem subject to zero internal forces during the motion. We consider exactly the same simulation setup described in Section 5.3 , with the only difference being that for the load distribution we use $G^{\star}$.

Different mass ratios in (6.23) result in different load distributions, which in turn affect the resulting minimal traversal time. We have performed the simulation for some values of the mass ratios. Table 6.1 shows these values and the obtained objective function for each case. The parameters related to the manipulators and the object and the other ones needed for the simulation are exactly same as those used in Section 5.3.

From the simulation setup described in Section 5.3, we can see that the first manipulator has a bit more power capabilities than the second manipulator, suggesting that assigning more load to the first manipulator may result in a smaller traversal time. From Table 6.1, it can be seen that among the different cases, the case where $m_{\mathrm{O}_{1}} / m=0.35, m_{\mathrm{O}_{2}} / m=0.65$ has the smallest minimal traversal time, and that assigning more load to the first manipulator does not improve the objective function. This is because power capability is not the only factor affecting the problem and other factors such as the object's path and orientation, dynamic parameters of the manipulators and the object, and the imposed constraints on the motion are also affecting the problem. Figures 6.5 and 6.6 show the resulting joint torques and velocities for both manipulators, respectively, when the mass 
ratios are set to $m_{\mathrm{O}_{1}} / m=0.35$ and $m_{\mathrm{O}_{2}} / m=0.65$. In this simulation, although the first manipulator has more power capabilities, it has heavier and larger links which require more torques. We have repeated the simulations for manipulators with the same size and mass of the links (with the other parameters of the simulation being unchanged), and still assigning more load to the first manipulator does not improve the minimal traversal time.

The objective function obtained for the case with $m_{\mathrm{O}_{1}} / m=0.35, m_{\mathrm{O}_{2}} / m=0.65$ is equal to $0.637 \mathrm{sec}$. From Table 6.1, it can be seen that there is a significant difference between this value and the objective functions obtained for the cases where all load is assigned to only one of the manipulators, i.e., $m_{O_{1}} / m=0$ or 1. This highlights the advantages of using cooperating manipulators for tasks involving path tracking.

In another scenario, in order to have a significant difference between power capabilities of the two manipulators and see how it affects the problem, we change the torque bounds for the first manipulator and set them to 310, 248, 124 N.m and $-310,-248,-124$ N.m for the upper and lower bounds, respectively. Here, the bounds are given for the first, second and third joints, respectively. The other parameters of the simulation are same as those used in Section 5.3. The resulting objective functions for different cases of mass ratios are given in Table 6.2. It can be seen that among the different cases, the case where $m_{\mathrm{O}_{1}} / m=0.55, m_{\mathrm{O}_{2}} / m=0.45$ has the smallest minimal traversal time. Unlike the first scenario, here assigning more load to the first manipulator than the second one, improves the objective function.

We have performed the simulations using the pseudo-inverses proposed by Verginis et al. [2019] and Erhart and Hirche [2015] to compare the resulting minimal traversal times with the ones obtained from our approach. Here, we report the results for the two scenarios discussed above. Using the pseudo-inverse proposed by Verginis et al. [2019] the results are $0.595 \mathrm{sec}$ and $0.538 \mathrm{sec}$ for the first and second scenarios, respectively. For Erhart and Hirche [2015], the minimal traversal times are $0.614 \mathrm{sec}$ and $0.505 \mathrm{sec}$ for the first and second scenarios, respectively. Using our proposed pseudo-inverse the results are $0.636 \mathrm{sec}$ and $0.527 \mathrm{sec}$ for the same scenarios, respectively. Compared to Verginis et al. [2019], our approach results in a slightly better minimal traversal time in the second scenario and a bit worse in the first scenario. Compared to Erhart and Hirche [2015], the results obtained by their proposed pseudo-inverse are slightly better in both scenarios. For further comparison between our work and Verginis et al. [2019], we have carried out simulations for four other scenarios. The details of the scenarios and the results are left out. In two of them our proposed pseudo-inverse gave smaller minimal traversal time and in the other two, the pseudo-inverse in Verginis et al. [2019] resulted in a smaller time. The improvements in all cases were less than 6\%. Again, for further comparison between our work and Erhart and Hirche [2015], we have performed simulations for four other scenarios. Their result was less than $5 \%$ smaller than our result in all four scenarios. One observation regarding the simulations using the proposed parameterized pseudo-inverse by Erhart and Hirche [2015] is that changing the parameters of the pseudo-inverse does not affect the resulting minimal traversal time 

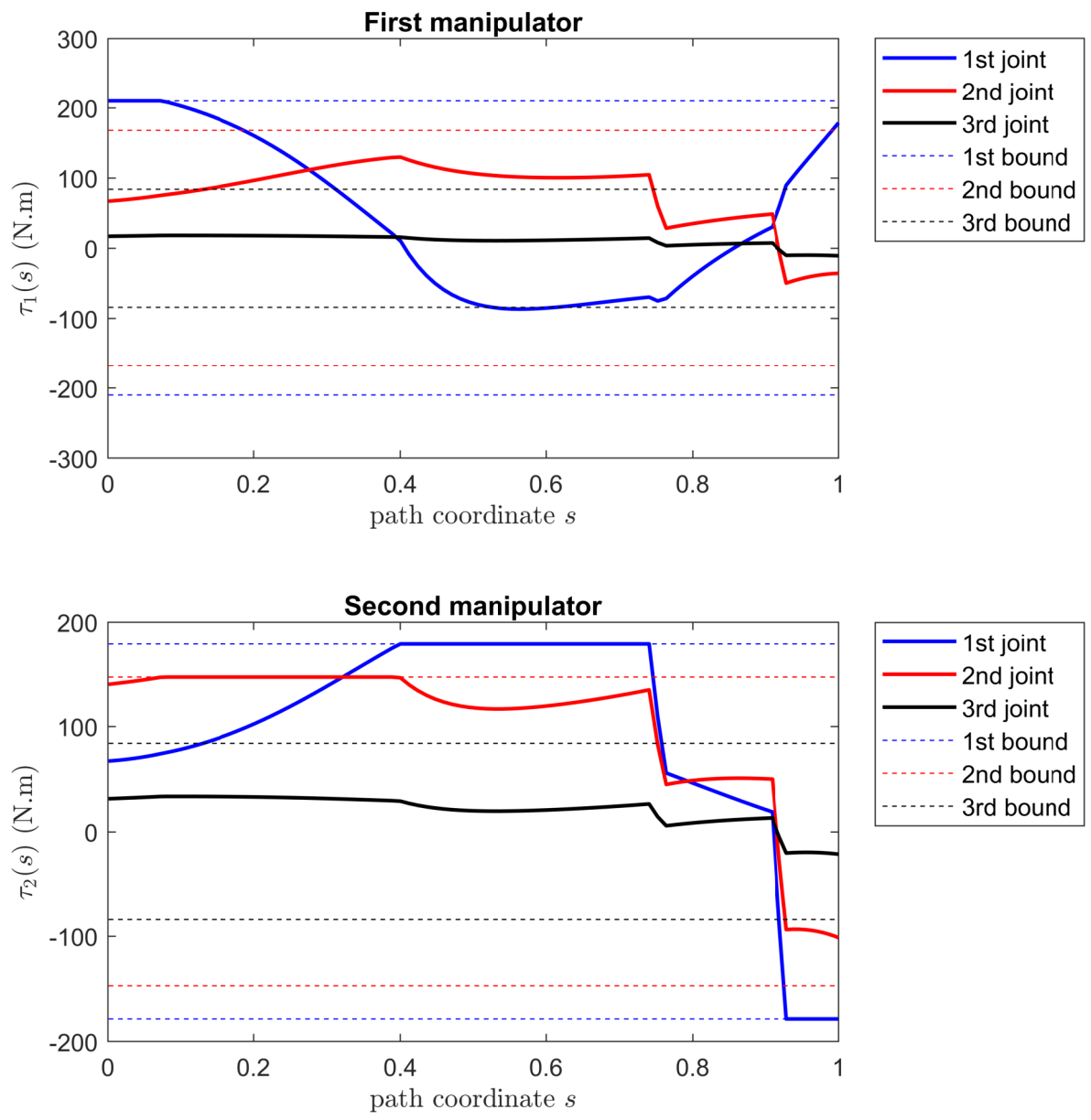

Figure 6.5: Joint torques $\tau_{1}(s)$ and $\tau_{2}(s)$. Dashed lines show the considered bounds on the joint torques. 

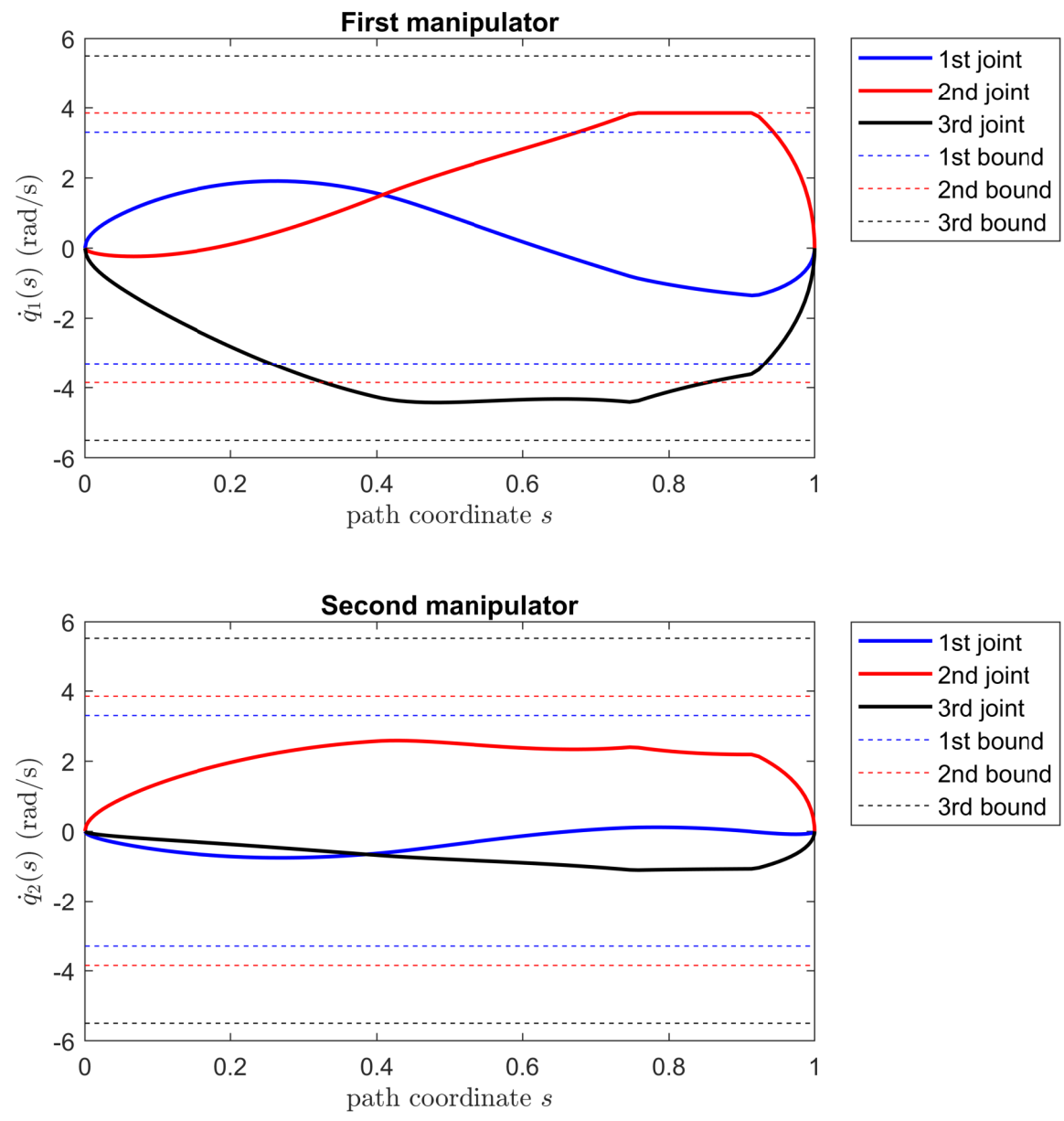

Figure 6.6: Joint velocities $\dot{q}_{1}(s)$ and $\dot{q}_{2}(s)$. Dashed lines show the considered bounds on the joint velocities. 
significantly and the results are very close to the optimal one (less than $2 \%$ difference). This is different from what is seen from the simulation results using our proposed parameterized pseudo-inverse. From the results in Tables 6.1 and 6.2 , it can be seen that changing the parameters of our proposed pseudo-inverse can result in a significant variation in the minimal traversal time. Different set of parameters in our approach mean different load distributions which expectedly affect the traversal time. This can be related to the fact that in the pseudo-inverse proposed by Erhart and Hirche [2015], the choice of the parameters related to mass is limited and the inertia-related parameters play a bigger role. 
Table 6.1: Obtained minimal traversal time for some values of the mass ratios.

\begin{tabular}{|c|c|c|}
\hline$m_{\mathrm{O}_{1}} / m$ & $m_{\mathrm{O}_{2}} / m$ & Minimal traversal time $(\mathrm{sec})$ \\
\hline 0 & 1 & 1.408 \\
\hline 0.15 & 0.85 & 0.855 \\
\hline 0.25 & 0.75 & 0.714 \\
\hline 0.35 & 0.65 & 0.637 \\
\hline 0.45 & 0.55 & 0.646 \\
\hline 0.5 & 0.5 & 0.661 \\
\hline 0.55 & 0.45 & 0.684 \\
\hline 0.65 & 0.35 & 0.751 \\
\hline 0.75 & 0.25 & 0.847 \\
\hline 0.85 & 0.15 & 1.001 \\
\hline 1 & 0 & 1.611 \\
\hline
\end{tabular}

Table 6.2: Obtained minimal traversal time for some values of the mass ratios for the case where the torque upper bounds for the first manipulator are set to $310,248,124$ N.m.

\begin{tabular}{|c|c|c|}
\hline$m_{\mathrm{O}_{1}} / m$ & $m_{\mathrm{O}_{2}} / m$ & Minimal traversal time $(\mathrm{sec})$ \\
\hline 0 & 1 & 1.408 \\
\hline 0.15 & 0.85 & 0.854 \\
\hline 0.25 & 0.75 & 0.714 \\
\hline 0.35 & 0.65 & 0.623 \\
\hline 0.45 & 0.55 & 0.556 \\
\hline 0.5 & 0.5 & 0.530 \\
\hline 0.55 & 0.45 & 0.527 \\
\hline 0.65 & 0.35 & 0.535 \\
\hline 0.75 & 0.25 & 0.551 \\
\hline 0.85 & 0.15 & 0.570 \\
\hline 1 & 0 & 0.617 \\
\hline
\end{tabular}





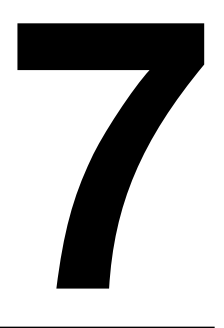

\section{Concluding remarks}

This chapter concludes the thesis by summarizing the results and discussing potential avenues for future work.

\subsection{Conclusions}

The overall objective of this thesis was to investigate the time-optimal path tracking problem for a team of cooperating robotic manipulators. The main contributions are:

- Formulating the time-optimal cooperative path tracking problem as a convex optimization problem and subsequently as an SOCP.

- Proposing a new approach for obtaining internal force-free load distributions.

The first contribution was covered in Chapters 4 and 5. Both chapters dealt with the time-optimal cooperative path tracking problem. The difference being that in Chapter 5, the problem of interest was addressed for a more general scenario comprised of generic manipulators and object. The approaches to formulate the problems of interest as convex optimization problems in two chapters involved using the first- and the second-order time derivatives of the path coordinate $s$ as optimization variables and deriving the coupled dynamics between the manipulators and the object. It was shown that using the aforementioned change of variables, the derived coupled dynamics becomes linear in optimization variables. In Chapter 5, the load distribution was determined via a generic pseudo-inverse of the grasp matrix. This allows for different possibilities. For example, the pseudo-inverse that was derived in Chapter 6 and results in internal force-free distributions can be used to impose this desired constraint on the 
motion. In the simulation studies in Chapters 4 and 5, it was seen that for every point along the path for the centre of mass of the object, either one of the joint torques of the first or second manipulator was in saturation, or one of the joint velocities had reached its bound. This is in line with the results that can be found in the literature about the structure of the minimum-time control for multiple cooperating manipulators.

The second contribution was covered in Chapter 6. In this chapter, the problem of finding a pseudo-inverse of the grasp matrix that results in internal forcefree distribution was addressed. A new approach to this problem was proposed and a new parameterized pseudo-inverse was derived. Compared to Erhart and Hirche [2015] which also proposes a parameterized pseudo-inverse, our approach is more intuitive since it is based on dividing the object into several segments and the parameter used for the load distributions have the meaning of the inertial parameters of these segments. In our approach it is clear how to assign more load to a particular manipulator, whereas in Erhart and Hirche [2015] it is not straightforward how to do this. A simulation study showed the use of this pseudo-inverse in the time-optimal cooperative path tracking problem where additionally it was required to have no internal forces during the motion. In the simulation study, the effect of assigning more load to one of the manipulators on the resulting minimal traversal time was discussed for different scenarios. It was seen that assigning more load to the manipulator with more power capability does not always improve the minimal traversal time. The reason is that factors such as the object's path and orientation, dynamic parameters of the manipulators and the object, and the imposed constraints on the motion are also affecting the problem and the optimal solution. Also, comparisons were made between the resulting minimal traversal times from our approach and the ones obtained using the proposed pseudo-inverses in Verginis et al. [2019] and Erhart and Hirche [2015]. In both comparisons, the results were approximately the same for the scenarios that were carried out.

\subsection{Future work}

The work presented in this thesis can be extended in several ways. One possible extension of the results in Chapter 5 is to incorporate constraints that do not preserve the convexity of the problem, such as velocity-dependent torque and torque rate constraints. Including this type of constraints can expand the applicability of the proposed procedure to more practical scenarios. Another possible direction for future work is to devise a distributed algorithm for solving the formulated optimization problem. Despite the success of centralized solvers for solving medium to large-scale problems, there are situations for which these solvers cannot be used. Issues such as limited computational power or memory, or privacy requirements can prevent us from using centralized solvers or even forming the problem in a centralized manner. In such situations, distributed algorithms might be used to solve the problem. Using distributed algorithms can also provide increased flexibility and modularity in the system. These algorithms 
solve the problem using a network of computational agents without the need for a centralized unit (Khoshfetrat Pakazad et al. [2017]).

Ideas for future work in the direction of the results presented in Chapter 6 include the incorporation of the freedom in the choice of the parameters of the pseudo-inverse as optimization variables into the problem. In the simulation study in Chapter 6, we explored this idea to a small degree by performing the simulation for each set of values of the parameters and comparing the resulting objective functions. This idea can be investigated in a more systematic way. The results in this chapter were explored for scenarios where the object is three-dimensional and convex and has uniform mass density. Another possible direction for future work is to extend these results to scenarios with nonconvex objects. 


\section{Bibliography}

Pro-act, robotic collaboration for isru. URL https://www . h2020-pro-act . eu/.

Technology research news. cooperative robots share the load. 2002. URL http://trnmag.com/Stories/2002/021302/Cooperative_robots_ share_the_load_021302.html.

MOSEK ApS. The MOSEK optimization toolbox for MATLAB manual. Version 9.2., 2021. URL http://docs.mosek.com/9.2/toolbox/index.html.

Tohid Ardeshiri, Mikael Norrlöf, Johan Löfberg, and Anders Hansson. Convex optimization approach for time-optimal path tracking of robots with speed dependent constraints. IFAC Proceedings Volumes, 44(1):14648-14653, 2011.

James E Bobrow, Steven Dubowsky, and John S Gibson. Time-optimal control of robotic manipulators along specified paths. The international journal of robotics research, 4(3):3-17, 1985.

James E Bobrow, J Michael McCarthy, and VK Chu. Minimum-time trajectories for two robots holding the same workpiece. In 29th IEEE Conference on Decision and Control, pages 3102-3107. IEEE, 1990.

Robert G Bonitz and Tien C Hsia. Force decomposition in cooperating manipulators using the theory of metric spaces and generalized inverses. In Proceedings of the 1994 IEEE International Conference on Robotics and Automation, pages 1521-1527. IEEE, 1994.

Stephen Boyd, Stephen P Boyd, and Lieven Vandenberghe. Convex optimization. Cambridge university press, 2004.

K-S Chang, Robert Holmberg, and Oussama Khatib. The augmented object model: Cooperative manipulation and parallel mechanism dynamics. In Proceedings 2000 ICRA. Millennium Conference. IEEE International Conference on Robotics and Automation. Symposia Proceedings (Cat. No. 00CH37065), volume 1, pages 470-475. IEEE, 2000. 
Yaobin Chen. Structure of the minimum-time control law for multiple robot arms handling a common object. In 1990 American Control Conference, pages 12861291. IEEE, 1990.

Yaobin Chen and Alan A Desrochers. Structure of minimum-time control law for robotic manipulators with constrained paths. In 1989 IEEE International Conference on Robotics and Automation, pages 971-972. IEEE Computer Society, 1989.

Howie M Choset, Kevin M Lynch, Seth Hutchinson, George Kantor, Wolfram Burgard, Lydia Kavraki, Sebastian Thrun, and Ronald C Arkin. Principles of robot motion: theory, algorithms, and implementation. MIT press, 2005.

Jae Heon Chung, Byung-Ju Yi, and Whee Kuk Kim. Analysis of internal loading at multiple robotic systems. Journal of mechanical science and technology, 19 (8):1554-1567, 2005.

Daniela Constantinescu and Elizabeth A Croft. Smooth and time-optimal trajectory planning for industrial manipulators along specified paths. Journal of robotic systems, 17(5):233-249, 2000.

Frederik Debrouwere and Jan Swevers. Conterweight synthesis for time-optimal robotic path following. In 2016 European Control Conference (ECC), pages 696-701. IEEE, 2016.

Frederik Debrouwere, Wannes Van Loock, Goele Pipeleers, Quoc Tran Dinh, Moritz Diehl, Joris De Schutter, and Jan Swevers. Time-optimal path following for robots with convex-concave constraints using sequential convex programming. IEEE Transactions on Robotics, 29(6):1485-1495, 2013.

Steven Dubowsky, M Norris, and Zvi Shiller. Time optimal trajectory planning for robotic manipulators with obstacle avoidance: a cad approach. In Proceedings. 1986 IEEE International Conference on Robotics and Automation, volume 3, pages 1906-1912. IEEE, 1986.

Sebastian Erhart and Sandra Hirche. Adaptive force/velocity control for multirobot cooperative manipulation under uncertain kinematic parameters. In 2013 IEEE/RSJ International Conference on Intelligent Robots and Systems, pages 307-314. IEEE, 2013.

Sebastian Erhart and Sandra Hirche. Internal force analysis and load distribution for cooperative multi-robot manipulation. IEEE Transactions on Robotics, 31 (5):1238-1243, 2015.

Sebastian Erhart and Sandra Hirche. Model and analysis of the interaction dynamics in cooperative manipulation tasks. IEEE Transactions on Robotics, 32 (3):672-683, 2016.

Michael Gienger, Dirk Ruiken, Tamas Bates, Mohamed Regaieg, Michael MeiBner, Jens Kober, Philipp Seiwald, and Arne-Christoph Hildebrandt. Human-robot 
cooperative object manipulation with contact changes. In 2018 IEEE/RSJ International Conference on Intelligent Robots and Systems (IROS), pages 13541360. IEEE, 2018.

Hamed Haghshenas, Mikael Norrlöf, and Anders Hansson. A convex optimization approach to time-optimal path tracking problem for cooperative manipulators. IFAC-PapersOnLine, 52(10):400-405, 2019.

John M Hollerbach. Dynamic scaling of manipulator trajectories. In 1983 American Control Conference, pages 752-756. IEEE, 1983.

Sina Khoshfetrat Pakazad, Anders Hansson, Martin S Andersen, and Isak Nielsen. Distributed primal-dual interior-point methods for solving tree-structured coupled convex problems using message-passing. Optimization Methods and Software, 32(3):401-435, 2017.

Vijay R Kumar and Kenneth J Waldron. Force distribution in closed kinematic chains. IEEE Journal on Robotics and Automation, 4(6):657-664, 1988.

Steven M LaValle. Planning algorithms. Cambridge university press, 2006.

Thomas Lipp and Stephen Boyd. Minimum-time speed optimisation over a fixed path. International Journal of Control, 87(6):1297-1311, 2014.

Johan Lofberg. Yalmip: A toolbox for modeling and optimization in matlab. In 2004 IEEE international conference on robotics and automation (IEEE Cat. No. 04CH37508), pages 284-289. IEEE, 2004.

Jeremy Maitin-Shepard, Marco Cusumano-Towner, Jinna Lei, and Pieter Abbeel. Cloth grasp point detection based on multiple-view geometric cues with application to robotic towel folding. In 2010 IEEE International Conference on Robotics and Automation, pages 2308-2315. IEEE, 2010.

Seungbin B Moon and Shaheen Ahmad. Time optimal trajectories for cooperative multi-robot systems. In 29th IEEE Conference on Decision and Control, pages 1126-1127. IEEE, 1990.

Seungbin B Moon and Shaheen Ahmad. Time scaling of cooperative multirobot trajectories. IEEE transactions on systems, man, and cybernetics, 21(4):900908, 1991.

Friedrich Pfeiffer and Rainer Johanni. A concept for manipulator trajectory planning. IEEE Journal on Robotics and Automation, 3(2):115-123, 1987.

Pedro Reynoso-Mora, Wenjie Chen, and Masayoshi Tomizuka. On the timeoptimal trajectory planning and control of robotic manipulators along predefined paths. In 2013 American Control Conference, pages 371-377. IEEE, 2013.

Kang Shin and Neil McKay. Minimum-time control of robotic manipulators with geometric path constraints. IEEE Transactions on Automatic Control, 30(6): $531-541,1985$. 
Bruno Siciliano and Oussama Khatib. Springer handbook of robotics. springer, 2016.

Bruno Siciliano, Lorenzo Sciavicco, Luigi Villani, and Giuseppe Oriolo. Robotics: modelling, planning and control. Springer Science \& Business Media, 2010.

J-JE Slotine and Hyun S Yang. Improving the efficiency of time-optimal pathfollowing algorithms. In 1988 American Control Conference, pages 2129-2134. IEEE, 1988.

Mark W Spong, Seth Hutchinson, Mathukumalli Vidyasagar, et al. Robot modeling and control, volume 3. wiley New York, 2006.

Armin Steinhauser and Jan Swevers. An efficient iterative learning approach to time-optimal path tracking for industrial robots. IEEE Transactions on Industrial Informatics, 14(11):5200-5207, 2018.

Anastasios Tsiamis, Christos K Verginis, Charalampos P Bechlioulis, and Kostas J Kyriakopoulos. Cooperative manipulation exploiting only implicit communication. In 2015 IEEE/RSJ International Conference on Intelligent Robots and Systems (IROS), pages 864-869. IEEE, 2015.

Christos K Verginis, Daniel Zelazo, and Dimos V Dimarogonas. Cooperative manipulation via internal force regulation: A rigidity theory perspective. arXiv preprint arXiv:1911.01297, 2019.

Diederik Verscheure, Bram Demeulenaere, Jan Swevers, Joris De Schutter, and Moritz Diehl. Time-optimal path tracking for robots: A convex optimization approach. IEEE Transactions on Automatic Control, 54(10):2318-2327, 2009.

Ian D Walker, Robert A Freeman, and Steven I Marcus. Analysis of motion and internal loading of objects grasped by multiple cooperating manipulators. The International journal of robotics research, 10(4):396-409, 1991. 


\section{Licentiate Theses \\ Division of Automatic Control \\ Linköping University}

P. Andersson: Adaptive Forgetting through Multiple Models and Adaptive Control of Car Dynamics. Thesis No. 15, 1983.

B. Wahlberg: On Model Simplification in System Identification. Thesis No. 47, 1985.

A. Isaksson: Identification of Time Varying Systems and Applications of System Identification to Signal Processing. Thesis No. 75, 1986.

G. Malmberg: A Study of Adaptive Control Missiles. Thesis No. 76, 1986.

S. Gunnarsson: On the Mean Square Error of Transfer Function Estimates with Applications to Control. Thesis No. 90, 1986.

M. Viberg: On the Adaptive Array Problem. Thesis No. 117, 1987.

K. Ståhl: On the Frequency Domain Analysis of Nonlinear Systems. Thesis No. 137, 1988.

A. Skeppstedt: Construction of Composite Models from Large Data-Sets. Thesis No. 149, 1988.

P. A. J. Nagy: MaMiS: A Programming Environment for Numeric/Symbolic Data Processing. Thesis No. 153, 1988.

K. Forsman: Applications of Constructive Algebra to Control Problems. Thesis No. 231, 1990.

I. Klein: Planning for a Class of Sequential Control Problems. Thesis No. 234, 1990.

F. Gustafsson: Optimal Segmentation of Linear Regression Parameters. Thesis No. 246, 1990.

H. Hjalmarsson: On Estimation of Model Quality in System Identification. Thesis No. 251, 1990.

S. Andersson: Sensor Array Processing; Application to Mobile Communication Systems and Dimension Reduction. Thesis No. 255, 1990.

K. Wang Chen: Observability and Invertibility of Nonlinear Systems: A Differential Algebraic Approach. Thesis No. 282, 1991.

J. Sjöberg: Regularization Issues in Neural Network Models of Dynamical Systems. Thesis No. 366, 1993.

P. Pucar: Segmentation of Laser Range Radar Images Using Hidden Markov Field Models. Thesis No. 403, 1993.

H. Fortell: Volterra and Algebraic Approaches to the Zero Dynamics. Thesis No. 438, 1994.

T. McKelvey: On State-Space Models in System Identification. Thesis No. 447, 1994.

T. Andersson: Concepts and Algorithms for Non-Linear System Identifiability. Thesis No. 448, 1994.

P. Lindskog: Algorithms and Tools for System Identification Using Prior Knowledge. Thesis No. 456, 1994.

J. Plantin: Algebraic Methods for Verification and Control of Discrete Event Dynamic Systems. Thesis No. 501, 1995.

J. Gunnarsson: On Modeling of Discrete Event Dynamic Systems, Using Symbolic Algebraic Methods. Thesis No. 502, 1995.

A. Ericsson: Fast Power Control to Counteract Rayleigh Fading in Cellular Radio Systems. Thesis No. 527, 1995.

M. Jirstrand: Algebraic Methods for Modeling and Design in Control. Thesis No. 540, 1996.

K. Edström: Simulation of Mode Switching Systems Using Switched Bond Graphs. Thesis No. 586, 1996. 
J. Palmqvist: On Integrity Monitoring of Integrated Navigation Systems. Thesis No. 600, 1997.

A. Stenman: Just-in-Time Models with Applications to Dynamical Systems. Thesis No. 601, 1997.

M. Andersson: Experimental Design and Updating of Finite Element Models. Thesis No. 611, 1997.

U. Forssell: Properties and Usage of Closed-Loop Identification Methods. Thesis No. 641, 1997.

M. Larsson: On Modeling and Diagnosis of Discrete Event Dynamic systems. Thesis No. 648, 1997.

N. Bergman: Bayesian Inference in Terrain Navigation. Thesis No. 649, 1997.

V. Einarsson: On Verification of Switched Systems Using Abstractions. Thesis No. 705, 1998.

J. Blom, F. Gunnarsson: Power Control in Cellular Radio Systems. Thesis No. 706, 1998.

P. Spångéus: Hybrid Control using LP and LMI methods - Some Applications. Thesis No. 724, 1998.

M. Norrlöf: On Analysis and Implementation of Iterative Learning Control. Thesis No. 727, 1998.

A. Hagenblad: Aspects of the Identification of Wiener Models. Thesis No. 793, 1999.

F. Tjärnström: Quality Estimation of Approximate Models. Thesis No. 810, 2000.

C. Carlsson: Vehicle Size and Orientation Estimation Using Geometric Fitting. Thesis No. 840, 2000.

J. Löfberg: Linear Model Predictive Control: Stability and Robustness. Thesis No. 866, 2001.

O. Härkegård: Flight Control Design Using Backstepping. Thesis No. 875, 2001.

J. Elbornsson: Equalization of Distortion in A/D Converters. Thesis No. 883, 2001.

J. Roll: Robust Verification and Identification of Piecewise Affine Systems. Thesis No. 899, 2001.

I. Lind: Regressor Selection in System Identification using ANOVA. Thesis No. 921, 2001.

R. Karlsson: Simulation Based Methods for Target Tracking. Thesis No. 930, 2002.

P.-J. Nordlund: Sequential Monte Carlo Filters and Integrated Navigation. Thesis No. 945, 2002.

M. Östring: Identification, Diagnosis, and Control of a Flexible Robot Arm. Thesis No. 948, 2002.

C. Olsson: Active Engine Vibration Isolation using Feedback Control. Thesis No. 968, 2002.

J. Jansson: Tracking and Decision Making for Automotive Collision Avoidance. Thesis No. 965, 2002.

N. Persson: Event Based Sampling with Application to Spectral Estimation. Thesis No. 981, 2002.

D. Lindgren: Subspace Selection Techniques for Classification Problems. Thesis No. 995, 2002.

E. Geijer Lundin: Uplink Load in CDMA Cellular Systems. Thesis No. 1045, 2003.

M. Enqvist: Some Results on Linear Models of Nonlinear Systems. Thesis No. 1046, 2003.

T. Schön: On Computational Methods for Nonlinear Estimation. Thesis No. 1047, 2003.

F. Gunnarsson: On Modeling and Control of Network Queue Dynamics. Thesis No. 1048, 2003.

S. Björklund: A Survey and Comparison of Time-Delay Estimation Methods in Linear Systems. Thesis No. 1061, 2003. 
M. Gerdin: Parameter Estimation in Linear Descriptor Systems. Thesis No. 1085, 2004.

A. Eidehall: An Automotive Lane Guidance System. Thesis No. 1122, 2004.

E. Wernholt: On Multivariable and Nonlinear Identification of Industrial Robots. Thesis No. 1131, 2004.

J. Gillberg: Methods for Frequency Domain Estimation of Continuous-Time Models. Thesis No. 1133, 2004.

G. Hendeby: Fundamental Estimation and Detection Limits in Linear Non-Gaussian Systems. Thesis No. 1199, 2005.

D. Axehill: Applications of Integer Quadratic Programming in Control and Communication. Thesis No. 1218, 2005.

J. Sjöberg: Some Results On Optimal Control for Nonlinear Descriptor Systems. Thesis No. 1227, 2006.

D. Törnqvist: Statistical Fault Detection with Applications to IMU Disturbances. Thesis No. 1258, 2006.

H. Tidefelt: Structural algorithms and perturbations in differential-algebraic equations. Thesis No. 1318, 2007.

S. Moberg: On Modeling and Control of Flexible Manipulators. Thesis No. 1336, 2007.

J. Wallén: On Kinematic Modelling and Iterative Learning Control of Industrial Robots. Thesis No. 1343, 2008.

J. Harju Johansson: A Structure Utilizing Inexact Primal-Dual Interior-Point Method for Analysis of Linear Differential Inclusions. Thesis No. 1367, 2008.

J. D. Hol: Pose Estimation and Calibration Algorithms for Vision and Inertial Sensors. Thesis No. 1370, 2008.

H. Ohlsson: Regression on Manifolds with Implications for System Identification. Thesis No. 1382, 2008.

D. Ankelhed: On low order controller synthesis using rational constraints. Thesis No. 1398, 2009.

P. Skoglar: Planning Methods for Aerial Exploration and Ground Target Tracking. Thesis No. 1420, 2009.

C. Lundquist: Automotive Sensor Fusion for Situation Awareness. Thesis No. 1422, 2009.

C. Lyzell: Initialization Methods for System Identification. Thesis No. 1426, 2009.

R. Falkeborn: Structure exploitation in semidefinite programming for control. Thesis No. 1430, 2010.

D. Petersson: Nonlinear Optimization Approaches to $\mathcal{H}_{2}$-Norm Based LPV Modelling and Control. Thesis No. 1453, 2010.

Z. Sjanic: Navigation and SAR Auto-focusing in a Sensor Fusion Framework. Thesis No. 1464, 2011.

K. Granström: Loop detection and extended target tracking using laser data. Thesis No. 1465, 2011.

J. Callmer: Topics in Localization and Mapping. Thesis No. 1489, 2011.

F. Lindsten: Rao-Blackwellised particle methods for inference and identification. Thesis No. 1480, 2011.

M. Skoglund: Visual Inertial Navigation and Calibration. Thesis No. 1500, 2011.

S. Khoshfetrat Pakazad: Topics in Robustness Analysis. Thesis No. 1512, 2011.

P. Axelsson: On Sensor Fusion Applied to Industrial Manipulators. Thesis No. 1511, 2011.

A. Carvalho Bittencourt: On Modeling and Diagnosis of Friction and Wear in Industrial Robots. Thesis No. 1516, 2012.

P. Rosander: Averaging level control in the presence of frequent inlet flow upsets. Thesis No. 1527, 2012. 
N. Wahlström: Localization using Magnetometers and Light Sensors. Thesis No. 1581, 2013.

R. Larsson: System Identification of Flight Mechanical Characteristics. Thesis No. 1599, 2013.

Y. Jung: Estimation of Inverse Models Applied to Power Amplifier Predistortion. Thesis No. 1605, 2013.

M. Syldatk: On Calibration of Ground Sensor Networks. Thesis No. 1611, 2013.

M. Roth: Kalman Filters for Nonlinear Systems and Heavy-Tailed Noise. Thesis No. 1613, 2013.

D. Simon: Model Predictive Control in Flight Control Design - Stability and Reference Tracking. Thesis No. 1642, 2014.

J. Dahlin: Sequential Monte Carlo for inference in nonlinear state space models. Thesis No. 1652, 2014.

M. Kok: Probabilistic modeling for positioning applications using inertial sensors. Thesis No. 1656, 2014.

J. Linder: Graybox Modelling of Ships Using Indirect Input Measurements. Thesis No. 1681, 2014.

G. Mathai: Direction of Arrival Estimation of Wideband Acoustic Wavefields in a Passive Sensing Environment. Thesis No. 1721, 2015.

I. Nielsen: On Structure Exploiting Numerical Algorithms for Model Predictive Control. Thesis No. 1727, 2015.

C. Veibäck: Tracking of Animals Using Airborne Cameras. Thesis No. 1761, 2016.

N. Evestedt: Sampling Based Motion Planning for Heavy Duty Autonomous Vehicles. Thesis No. 1762, 2016.

H. Nyqvist: On Pose Estimation in Room-Scaled Environments. Thesis No. 1765, 2016.

Y. Zhao: Position Estimation in Uncertain Radio Environments and Trajectory Learning. Thesis No. 1772, 2017.

P. Kasebzadeh: Parameter Estimation for Mobile Positioning Applications. Thesis No. 1786, 2017.

K. Radnosrati: On Timing-Based Localization in Cellular Radio Networks. Thesis No. 1808, 2018.

G. Lindmark: Methods and Algorithms for Control Input Placement in Complex Networks. Thesis No. 1814, 2018.

M. Lindfors: Frequency Tracking for Speed Estimation. Thesis No. 1815, 2018.

D. Ho: Some results on closed-loop identification of quadcopters. Thesis No. 1826, 2018.

O. Ljungqvist: On motion planning and control for truck and trailer systems. Thesis No. 1832, 2019.

P. Boström-Rost: On Informative Path Planning for Tracking and Surveillance. Thesis No. 1838, 2019.

K. Bergman: On Motion Planning Using Numerical Optimal Control. Thesis No. 1843, 2019.

M. Klingspor: Low-rank optimization in system identification. Thesis No. 1855, 2019.

A. Bergström: Timing-Based Localization using Multipath Information. Thesis No. 1867, 2019.

F. Ljungberg: Estimation of Nonlinear Greybox Models for Marine Applications. Thesis No. 1880, 2020.

E. Hedberg: Control, Models and Industrial Manipulators. Thesis No. 1894, 2020.

R. Forsling: Decentralized Estimation Using Conservative Information Extraction. Thesis No. 1897, 2020. 
D. Arnström: On Complexity Certification of Active-Set QP Methods with Applications to Linear MPC. Thesis No. 1901, 2021.

M. Malmström: Uncertainties in Neural Networks: A System Identification Approach. Thesis No. 1902, 2021.

K. Nielsen: Robust LIDAR-Based Localization in Underground Mines. Thesis No. 1906, 2021. 


\section{FACULTY OF SCIENCE AND ENGINEERING}

Linköping studies in science and technology. Licentiate Thesis No. 1915, 2021 Department of Electrical Engineering

Linköping University

SE-581 83 Linköping, Sweden

www.liu.se 